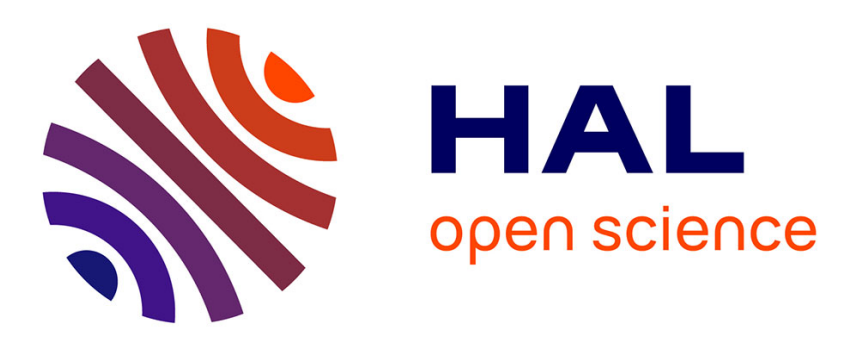

\title{
Shape optimization with Stokes constraints over the set of axisymmetric domains
}

Maïtine Bergounioux, Yannick Privat

\section{To cite this version:}

Maïtine Bergounioux, Yannick Privat. Shape optimization with Stokes constraints over the set of axisymmetric domains. SIAM Journal on Control and Optimization, 2013, 51 (1), pp.599-628. $10.1137 / 100818133$. hal-00546165v2

\section{HAL Id: hal-00546165 \\ https://hal.science/hal-00546165v2}

Submitted on 1 Nov 2011

HAL is a multi-disciplinary open access archive for the deposit and dissemination of scientific research documents, whether they are published or not. The documents may come from teaching and research institutions in France or abroad, or from public or private research centers.
L'archive ouverte pluridisciplinaire HAL, est destinée au dépôt et à la diffusion de documents scientifiques de niveau recherche, publiés ou non, émanant des établissements d'enseignement et de recherche français ou étrangers, des laboratoires publics ou privés. 


\title{
SHAPE OPTIMIZATION WITH STOKES CONSTRAINTS OVER THE SET OF AXISYMMETRIC DOMAINS*
}

\author{
MAITINE BERGOUNIOUX ${ }^{\dagger}$ AND YANNICK PRIVAT ${ }^{\ddagger}$
}

\begin{abstract}
In this paper, we are interested in the study of shape optimizations problems with Stokes constraints within the class of axisymmetric domains represented by the graph of a function. Existence results with weak assumptions on the regularity of the graph are provided. We strongly use these assumptions to get some topological properties. We formulate the (shape) optimization problem using different constraints formulations: uniform bound constraints on the function and its derivative and/or volume (global) constraint. Writing the first order optimality conditions allows to provide quasi-explicit solutions in some particular cases and to give some hints for the treatment of the generic problem. Furthermore, we extend the (negative) result of [16] dealing with the non optimality of the cylinder.
\end{abstract}

Key words. Shape optimization, Dissipated energy, Stokes system, First order optimality conditions.

AMS subject classifications. 49Q10, 49J20, 49K20, 35Q30, 76D05, 76D55

1. Introduction. The applications of shape optimization to fluid mechanics are numerous. Some well-known and studied situations may be encountered in industry for instance airplane optimization, where the drag is often minimized under a lift constraint (see e.g. [6, 2, 22, 26] for examples of such applied studies). More generally, the study of shape optimization problems in the context of fluid mechanics constitutes a challenge. Most of time, works on this topic are numerical point of view studies, because of the intrinsic difficulty of Stokes or Navier-Stokes equations. Among the well known and studied problems of shape optimization for fluids, one can mention, for instance, the reduction of the drag of an airplane wing in order to ensure hydrodynamic stability or the minimization of the noise of vortex shedding for designing the shape of an airfoil trailing edge.

The partial differential equation describing the behavior of the fluid appears then as an additional constraint for the optimization problem. For first references on this topic, we refer to $[12,14,26,29,30]$.

In $[15,16]$, a theoretical study on the shape minimization of the dissipated energy in a pipe has been led. In particular, the first order optimality conditions for the optimization problem were written and exploited to prove that, under some given particular boundary conditions chosen to model trachea in human beings, the cylinder is not an optimal solution. Nevertheless, some numerical computations done in the same papers let us think that the optimum is very close to a cylinder.

This paper is motivated by a simple question coming from the conclusions of [15]: indeed, it was proven that the cylinder does not optimize the dissipated energy through a pipe, when the fluid inside is driven by Stokes or Navier-Stokes laws. Moreover, the problem of knowing if the optimal solution has or does not have a cylindrical symmetry is pointed out and still open. Another point of view consists in imposing the cylindrical symmetry in the class of admissible shapes for this optimization problem. Such a choice can be justified by the fact that, in some situations, it is natural to make this assumption. For instance, if we assume that the shape of the (human being) trachea minimizes the energy dissipated by the air through the geometry (thanks to a natural selection process), it is reasonable to consider only simply connected domains with

\footnotetext{
*The second author was partially supported by ANR Project GAOS (Geometric Analysis of Optimal Shapes.

†Univ. d'Orléans, Labo. MAPMO, CNRS, UMR 6628, Fédération Denis Poisson, FR 2964, Bat. Math., BP 6759, 45067 Orléans cedex 2, France; maitine.bergounioux@univ-orleans.fr

‡ENS Cachan Bretagne, CNRS, Univ. Rennes 1, IRMAR, av. Robert Schuman, F-35170 Bruz, France; yannick.privat@bretagne.ens-cachan.fr
} 
cylindrical symmetry. With such a restriction on admissible shapes, we may hope simplifications of the system driving the behavior of the fluid to obtain existence of optimal shape results more easily and a simple expression of the first order optimality conditions. In this paper, we decided to make the (strong) hypothesis that a transversal slice of an admissible domain is the graph of a function $z \mapsto a(z)$. Our goal is to investigate the question of the existence of optimal shapes over the class of such cylindrical domains, with Stokes partial differential equations constraints and write the first order optimality conditions. This study may be seen as a preliminary study specially in view of a refined study of the qualitative properties of the optimum (for instance, the very difficult question of the free boundary regularity) and numerical computations.

As it will be emphasized in the following sections, it is quite easy to prove the existence of an optimal shape for three dimensional domains and to ensure a strong convergence of the terms of the minimizing sequence of domains to the optimum. Nevertheless, the applications, theoretical and numerical, previously mentioned need a precise frame adapted to the domains having a cylindrical symmetry. One of the difficult parts of our work lies in the determination of a variational formulation taking into account the cylindrical character of the domain and the symmetry properties of the solution of Stokes partial differential equations.

The paper is organized as follows: next section is devoted to optimal shape existence. We use the cylindrical symmetry assumption to give a 2D formulation of Stokes equation, using cylindrical coordinates. We use a fictitious domain technique to get result without strong regularity assumptions on the shape boundary. Proofs are given in Section 3. Optimality conditions are investigated in the last section. We first give a generic abstract result, and then a specific result in the "axisymmetric graph" case. If no volume constraint is added, we prove a generic monotonicity result of the cost functional for the inclusion of domains. As a result, the problem becomes purely geometrical which allows to provide a precise characterization of the optimum. The same shape optimization problem with an additional volume constraint appears rather difficult. We are nevertheless in position to establish the non optimality of the cylinder in that case (extending by the same the results of [16]). Moreover, we propose some hints to write the first order optimality conditions and prepare a future numerical work on that topic.

\section{Some shape existence results.}

2.1. Preliminaries. Let $L, a_{0}$ and $a_{1}$, be three strictly positive real numbers such that $a_{0}<a_{1}$. Let us introduce the set of admissible parametrizations

$$
\mathcal{A}_{\infty}=\left\{a \in W^{1, \infty}(0, L) \mid a_{0} \leq a(z) \leq a_{1} \text { for } z \in(0, L)\right\} .
$$

We consider a generic domain $\Omega_{a}$, assumed for the moment simply connected, bounded, with Lipschitz boundary and axisymmetric with respect to the $(O z)$-axis. More precisely, the domain expressed in standard cylindrical coordinates is

$$
\Omega_{a}=\left\{(r, \theta, z) \in \mathbb{R}_{+} \times \mathbb{T} \times \mathbb{R}_{+} \mid 0<r<a(z), 0<z<L\right\},
$$

where $\mathbb{T}$ denotes the torus $\mathbb{R} / 2 \pi$ and $a \in \mathcal{A}_{\infty}$. We denote by $\mathcal{D}_{a}$, the bounded open set, whose closure is

$$
\overline{\mathcal{D}_{a}}=\left\{(z, r) \in \mathbb{R}_{+} \times \mathbb{R}_{+} \mid 0 \leq z \leq L, 0 \leq r \leq a(z)\right\},
$$

so that $\Omega_{a}$ is obtained by a rotation of $\mathcal{D}_{a}$ around the axis $\{r=\theta=0\}$ denoted from now $(O z)$. We write

$$
\partial \Omega_{a}=E_{a} \cup \Sigma_{a} \cup S_{a},
$$

where $E_{a}=\overline{\Omega_{A}} \cup\{z=0\}$ is the inlet surface, $S_{a}=\overline{\Omega_{A}} \cap\{z=L\}$ is the outflow surface and $\Sigma_{a}=\partial \Omega_{a} \backslash\left(E_{a} \cup S_{a}\right)$ is the lateral surface. Similarly, we write $\partial \mathcal{D}_{a}=\Gamma_{0} \cup \Gamma_{L} \cup \Gamma_{b} \cup \Gamma_{a}$, where 
$\Gamma_{0}=\{(0, r) \mid 0 \leq r \leq a(0)\}, \Gamma_{L}=\{(L, r) \mid 0 \leq r \leq a(L)\}, \Gamma_{b}=\{(z, 0) \mid 0 \leq z \leq L\}$, $\Gamma_{a}=\{(z, a(z)) \mid 0 \leq z \leq L\}$. The notations used are summarized on Figure 2.1.
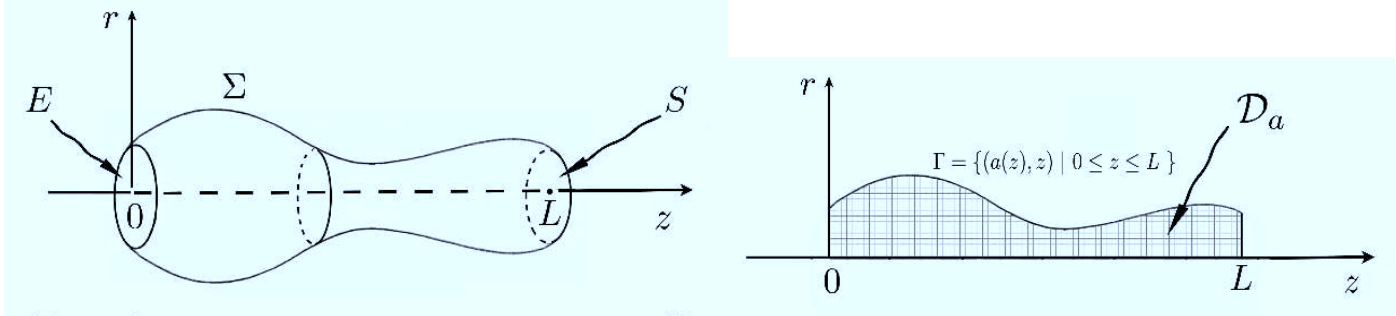

FIG. 2.1. A cylindrical admissible domain and its slice in two dimensions

The same model of fluid as in $[15,16]$ is studied, for instance to model the flow of the air inside the trachea represented by $\mathcal{D}_{a}$. More precisely, denoting by $\mathbf{u}$ the velocity of the fluid, $p$ its pressure, the Stokes system is written as

$$
\left\{\begin{aligned}
-\mu \Delta \mathbf{u}+\nabla p=0 & \text { in } \Omega_{a} \\
\nabla \cdot \mathbf{u}=0 & \text { in } \Omega_{a} \\
\mathbf{u}=0 & \text { on } \Sigma_{a} \\
\mathbf{u}=\mathbf{u}_{\mathbf{0}} & \text { on } E_{a} \\
\sigma(\mathbf{u}, p) \cdot \mathbf{n}=\mathbf{h} & \text { on } S_{a}
\end{aligned}\right.
$$

where $\mu>0$ stands for the viscosity of the fluid, $\mathbf{u}_{\mathbf{0}}$ is a Dirichlet datum, $\mathbf{h}$ a Neumann like datum which will be made precise later, and where the standard tensorial notations of fluid mechanics are used, i.e.

$$
\sigma(\mathbf{u}, p)=-p I_{3}+2 \mu \varepsilon(\mathbf{u})
$$

is the stress tensor of $(\mathbf{u}, p)$ and

$$
\varepsilon(\mathbf{u})=\frac{1}{2}\left(\nabla \mathbf{u}+(\nabla \mathbf{u})^{\top}\right)=\left(\frac{1}{2}\left(\frac{\partial u_{i}}{\partial x_{j}}+\frac{\partial u_{j}}{\partial x_{i}}\right)\right)_{1 \leq i, j \leq 3},
$$

is the strain tensor of $\mathbf{u}$ (symmetric part of the gradient tensor). We also define the trace inner product of two vector fields $\mathbf{A}$ and $\mathbf{B}$ of $\mathbb{R}^{d}, d=2,3$ by

$$
\mathbf{A}: \mathbf{B}=\sum_{i, j=1}^{d} A_{i j} B_{i j}
$$

In the whole paper, the bold letters stand for vector fields of $\mathbb{R}^{2}$ or $\mathbb{R}^{3}$.

The existence of solutions for System (2.4) is well known (see for instance [3, 13]).

Theorem 2.1. Let $a \in \mathcal{A}_{\infty}$. Assume that $\mathbf{u}_{\mathbf{0}} \in\left(H^{3 / 2}\left(E_{a}\right)\right)^{3}, \mathbf{h} \in\left(H^{1 / 2}\left(S_{a}\right)\right)^{3}$, then problem (2.4) has a unique solution $(\mathbf{u}, p) \in\left(H^{1}\left(\Omega_{a}\right)\right)^{3} \times L^{2}\left(\Omega_{a}\right)$.

Since we are dealing with stokes equations, we have to assume that $a$ (roughly speaking the boundary of the domain) is smooth enough (say for instance $a \in \mathcal{A}_{\infty}$ ) to get regular solutions. The existence and regularity of solutions to Stokes equations in non smooth domains has not been investigated so much. Let us nevertheless mention the works [7, 8, 24, 25, 32]. 
The assumption that $a \in \mathcal{A}_{\infty}$ will be relaxed in the sequel using a weak variational formulation to define solutions of (2.4).

For the inlet boundary condition, we will choose $\mathbf{u}_{\mathbf{0}}=\left(0,0, u_{0}(r)\right)^{\top}$, where $u_{0}$ is a positive function of the polar variable $r=\sqrt{x_{1}^{2}+x_{2}^{2}}$.

For the outlet boundary condition, we will choose in the sequel of the paper $\mathbf{h}=-p_{0} \mathbf{n}$, where $\mathbf{n}$ denotes the outward-pointing normal vector, and $p_{0}>0$ is a real number. In particular, this boundary condition can model the human bronchial tree (see e.g. [23]). With such a choice, introducing $\bar{p}=p-p_{0}$, that is easy to see that the pair $(\mathbf{u}, \bar{p})$ is solution of System $(2.4)$, where $\mathbf{h}$ has been replaced by 0 in the boundary condition on $S_{a}$. For this reason, we will actually choose $\mathbf{h}=0$ in the sequel.

Then, it is relevant to wonder whether we are able to rewrite the Stokes problem (2.4) using only cylindrical coordinates $(r, z)$, as we can easily have this intuition, since the geometry is cylindrically symmetric. Moreover, the functional we want to minimize is the energy dissipated by the fluid (or viscosity energy) defined by

$$
J_{0}(\Omega)=2 \mu \int_{\Omega}|\varepsilon(\mathbf{u})|^{2} d x,
$$

where $\mathbf{u}$ is the solution of System (2.4), in a certain class of admissible shapes parametrized for instance by the elements of $\mathcal{A}_{\infty}$.

We end this subsection with a standard, but essential ingredient for the coming existence study. The solution of the Stokes problem (2.4) can be seen as the unique minimizer of an energy functional $j$. We recall this fact and its proof for the sake of completeness.

LEMMA 2.2. $\mathbf{u}_{\boldsymbol{\Omega}_{\mathbf{a}}}$ is the (unique) solution of (2.4) if, and only if $\mathbf{u}_{\boldsymbol{\Omega}_{\mathbf{a}}}$ is a solution of

$$
\left\{\begin{array}{l}
\min j(\mathbf{u})=2 \mu \int_{\Omega_{a}}|\varepsilon(\mathbf{u})|^{2} d x \\
\mathbf{u} \in H_{\mathrm{div}}\left(\Omega_{a}\right)=\left\{\mathbf{v} \in\left[H^{1}\left(\Omega_{a}\right)\right]^{3} \mid \nabla \cdot \mathbf{v}=0 \text { in } \Omega_{a}, \mathbf{v}=0 \text { on } \Sigma \text { and } \mathbf{v}=\left(0,0, \mathbf{u}_{\mathbf{0}}\right) \text { on } E\right\} .
\end{array}\right.
$$

Proof. Let $\left(\mathbf{u}_{\mathbf{n}}\right)_{n \in \mathbb{N}}$ be a minimizing sequence of the optimization problem (2.6). Notice that, by virtue of Korn's inequality combined together with a Poincarés inequality (see [3]), the standard $\left[H^{1}\left(\Omega_{a}\right)\right]^{3}$-norm is equivalent to the norm $\|\cdot\|_{\varepsilon}$ induced by the inner product

$$
\langle\mathbf{f}, \mathbf{g}\rangle_{\varepsilon}=\int_{\Omega} \varepsilon(\mathbf{f}): \varepsilon(\mathbf{g}) d x
$$

where $\mathbf{f}$ and $\mathbf{g}$ denote two regular vector fields having the same dimension as $\Omega$.

The sequence $\left(j\left(\mathbf{u}_{\mathbf{n}}\right)\right)_{n \in \mathbb{N}}$ is bounded, and thus, there exists $\mathbf{u}^{\star} \in\left[H^{1}\left(\Omega_{a}\right)\right]^{3}$ such that

$$
\mathbf{u}_{\mathbf{n}} \stackrel{L^{2}(\Omega)}{\rightarrow} \mathbf{u}^{\star} \text { and } \mathbf{u}_{\mathbf{n}} \stackrel{H^{1}(\Omega)}{\rightarrow} \mathbf{u}^{\star} \text {, as } n \rightarrow+\infty \text {. }
$$

Moreover,

$$
\left\|\mathbf{u}^{\star}\right\|_{\varepsilon} \leq \liminf _{n \rightarrow+\infty}\left\|\mathbf{u}_{\mathbf{n}}\right\|_{\varepsilon}
$$

which proves the existence of a minimizer. To characterize it, we will use the standard De Rham's lemma (see e.g. [33]), stipulating that to take into account the divergence pointwise constraints, it is enough to work with test functions chosen in the space

$$
\mathcal{V}(\Omega)=\left\{\mathbf{w} \in \mathcal{D}\left(\Omega_{a}\right) ; \nabla \cdot \mathbf{w}=0 \text { in } \Omega_{a}\right\},
$$


where $\mathcal{D}\left(\Omega_{a}\right)$ stands for the set of $C^{\infty}(\Omega)$-functions having compact support in $\Omega_{a}$. Let $\mathbf{w} \in \mathcal{V}\left(\Omega_{a}\right)$. One has, by virtue of a well-known intergration by parts formula (see later Formula (3.9))

$$
\begin{aligned}
\lim _{t \rightarrow 0} \frac{j\left(\mathbf{u}^{\star}+t \mathbf{w}\right)-j\left(\mathbf{u}^{\star}\right)}{t} & =\int_{\Omega} \varepsilon\left(\mathbf{u}^{\star}\right): \varepsilon(\mathbf{w}) d x \\
& =-\mu \int_{\Omega}\left(\Delta \mathbf{u}^{\star}+\nabla\left(\nabla \cdot \mathbf{u}^{\star}\right)\right) \cdot \mathbf{w} d x
\end{aligned}
$$

The use of De Rham's lemma proves the existence of $p^{\star} \in L^{2}\left(\Omega_{a}\right)$ such that $\mathbf{u}^{\star}$ satisfies in the sense of distributions $-\mu \Delta \mathbf{u}^{\star}+\nabla p^{\star}=0$. The boundary conditions are derived as usual, using the variational formulation obtained thanks to De Rham's lemma.

2.2. An abstract shape existence result in dimension 3. To prove an existence result, we need to make the class of admissible domains precise. We denote by $D$ the cylindrical box defined by $D=\left\{\left(x_{1}, x_{2}, x_{3}\right) \in \mathbb{R}^{3} \mid x_{1}^{2}+x_{2}^{2} \leq R_{0}^{2}, 0 \leq x_{3} \leq L\right\}$, with $R_{0}>0$. Imposing some kind of regularity condition is a very classical feature in shape optimization, since these problems are often ill-posed, see $[1,14]$. We will consider quasi-open sets included in the string $B=\left\{\left(x_{1}, x_{2}, x_{3}\right) \in \mathbb{R}^{3} \mid 0 \leq x_{3} \leq L\right\}$. Let us recall that a subset $\Omega \subset D$ is said to be quasi-open if there exists a nonincreasing sequence of open sets $\left(\omega_{n}\right)_{n \in \mathbb{N}}$ such that

$$
\lim _{n \rightarrow+\infty} \operatorname{cap}\left(\omega_{n}\right)=0 \text { and } \forall n \in \mathbb{N}, \Omega \cup \omega_{n} \text { is an open set, }
$$

where cap denotes the standard capacity defined for compact or open sets (see e.g. [4, 14]). We fix $E \subset\left\{x_{3}=0\right\}$, a disk whose center is crossed by the axis $\left\{x_{1}=x_{2}=0\right\}$ and define

$$
\begin{aligned}
\mathcal{O}= & \{\Omega \text { quasi-open included in } D ; \\
& \left.\exists \mathbf{w} \in\left[H^{1}(D)\right]^{3} \text { with } \Omega=\{\mathbf{w} \neq 0\}, \mathbf{w}_{\left.\right|_{E}}=\left(0,0, u_{0}\right) \text { and } \operatorname{cap}\left(\bar{\Omega} \cap\left\{x_{3}=L\right\}\right) \neq 0\right\} .
\end{aligned}
$$

THEOREM 2.3. The problem

$$
\left\{\begin{array}{l}
\min J_{0}(\Omega)=2 \mu \int_{\Omega}\left|\varepsilon\left(\mathbf{u}_{\boldsymbol{\Omega}}\right)\right|^{2} d x \\
\Omega \in \mathcal{O} \\
|\Omega| \leq V_{0} \\
\mathbf{u}_{\Omega} \text { solution of }(2.4)
\end{array}\right.
$$

has (at least) one solution, whose volume may be chosen equal to $V_{0}$.

Proof. Let $\left(\Omega_{n}\right)_{n \in \mathbb{N}}$, be a minimizing sequence. Let us denote by $m$ the infimum. By virtue of Korn's inequality combined with a Poincaré like inequality (see [3]), and since all the admissible domains are contained in a compact set $D$, we know that $\mathbf{u}_{\mathbf{n}}=\mathbf{u}_{\Omega_{\mathbf{n}}}$ is bounded in $\left[H^{1}(D)\right]^{3}$ (indeed $\varepsilon\left(\mathbf{u}_{\boldsymbol{\Omega}_{\mathbf{n}}}\right)$ is $L^{2}$-bounded). Consequently, there exists $\mathbf{u}^{\star} \in\left[H^{1}(D)\right]^{3}$ such that $\left(\mathbf{u}_{\mathbf{n}}\right)$ converges to $\mathbf{u}^{\star}$ up to an extraction, weakly in $\left[H^{1}(D)\right]^{3}$ and strongly in $\left[L^{2}(D)\right]^{3}$. Using a compactness property of the trace in $\left[L^{2}(E)\right]^{3}$, the condition $\mathbf{u}=\left(0,0, u_{0}\right)$ is preserved on $E$. It remains to prove that $\operatorname{cap}\left(\overline{\Omega_{\mathbf{u}^{\star}}} \cap\left\{x_{3}=L\right\}\right) \neq 0$. For that purpose, let us use the fact that $\mathbf{u}_{\boldsymbol{\Omega}_{\mathbf{n}}}$ is solution of (2.4). Integrating the "divergence-free" condition on $\Omega_{\mathbf{u}_{\mathbf{n}}}$ yields

$$
-\int_{\text {Supp }\left(u_{0}\right)} u_{0} d s=\int_{D \cap\left\{x_{3}=L\right\}} u_{3, n} d s .
$$

Using the convergence results established previously and a compactness property of the trace, one gets that the above equality remains valid for $\mathbf{u}^{\star}$ and hence, ensures that $\Omega_{\mathbf{u}^{*}}$ belongs to $\mathcal{O}$. 
Then, the quasi-open $\Omega_{\mathbf{u}^{\star}}=\left\{\mathbf{u}^{\star} \neq 0\right\}$ belongs to $\mathcal{O}$. Furthermore, since $\mathbf{u}^{\star}=0$ quasieverywhere on $D \backslash \Omega_{\mathbf{u}^{\star}}$, by weak $H^{1}$-convergence, one has, by Lemma 2.6,

$$
\int_{\Omega_{\mathbf{u}^{\star}}}\left|\varepsilon\left(\mathbf{u}^{\star}\right)\right|^{2} d x \leq \liminf _{n \rightarrow+\infty} \int_{\Omega_{n}}\left|\varepsilon\left(\mathbf{u}_{\mathbf{n}}\right)\right|^{2} d x=m \leq \int_{\Omega_{\mathbf{u}^{\star}}}\left|\varepsilon\left(\mathbf{u}_{\mathbf{\Omega}_{\mathbf{u}^{\star}}^{\star}}\right)\right|^{2} d x \leq \int_{\Omega_{\mathbf{u}^{\star}}}\left|\varepsilon\left(\mathbf{u}^{\star}\right)\right|^{2} d x,
$$

whence the equality of these quantities.

Finally, thanks to the almost everywhere convergence of $\mathbf{u}_{\mathbf{n}}$ to $\mathbf{u}^{\star}$, one has also

$$
\left|\Omega_{\mathbf{u}^{\star}}\right| \leq \liminf _{n \rightarrow+\infty}\left|\Omega_{n}\right| \leq V_{0}
$$

Now, if $\left|\Omega_{\mathbf{u}^{\star}}\right|=V_{0}$, then $\widehat{\Omega}:=\Omega_{\mathbf{u}^{\star}}$ is solution of (2.7). If $\left|\Omega_{\mathbf{u}^{\star}}\right|<V_{0}$, let us consider any quasi-open $\widehat{\Omega}$ such that

$$
\Omega_{\mathbf{u}^{\star}} \subset \widehat{\Omega},|\widehat{\Omega}|=V_{0}
$$

Thanks to Lemma 2.2, rewriting the objective function $J_{0}$ under energetic form

$$
J_{0}(\Omega)=2 \mu \min \left\{j(\mathbf{u}), \mathbf{u} \in H_{\operatorname{div}}\left(\Omega_{a}\right)\right\}
$$

shows immediately that $J_{0}$ is a decreasing function with respect to the inclusion of sets. By monotonicity of this functional, one has $\int_{\widehat{\Omega}}\left|\varepsilon\left(\mathbf{u}_{\widehat{\Omega}}\right)\right|^{2} \leq \int_{\Omega_{\mathbf{u}^{\star}}}\left|\varepsilon\left(\mathbf{u}_{\boldsymbol{\Omega}_{\mathbf{u}^{\star}}^{\star}}\right)\right|^{2}$, which proves that $\widehat{\Omega}$ is solution of $(2.7)$.

REMARK 1. The solution is a priori not unique and we have to set additional "physical" constraints on the domain to get an acceptable solution from the physical point of view. That is why we embedded the problem in the class of cylindrical domains so that we may expect (in particular) uniqueness and more regularity of the optimal graph. Moreover, the choice of admissible shapes we will make in next section will simplify the study of the related optimization problem and to characterize quite precisely the solution.

2.3. Symmetry of solutions for Stokes problems in cylindrical domains. Before regarding the question of the existence for the shape optimization problem with Stokes constraint, over the set of axisymmetric domains, we need to point out some symmetry properties of the solutions of the Stokes system (2.4). We associate to the classical Cartesian coordinates $\left(x_{1}, x_{2}, x_{3}\right)$, the cylindrical coordinates, denoted $(r, \theta, z)$, defined by

$$
r=\sqrt{x_{1}^{2}+x_{2}^{2}}, \theta=\left\{\begin{array}{ll}
2 \arctan \left(\frac{x_{2}}{x_{1}+\sqrt{x_{1}^{2}+x_{2}^{2}}}\right) & \text { if }\left(x_{1}, x_{2}\right) \notin \mathbb{R}_{-} \times\{0\} \\
\pi & \text { else }
\end{array} \text { and } z=x_{3} .\right.
$$

Proposition 2.4. Let $a \in \mathcal{A}_{\infty}$. Let us assume that $u_{0}$ only depends on the variable $r$. Then, the solution $(\mathbf{u}, p)$ of (2.4) posed on $\Omega_{a}$ satisfies

1. $u_{3}$ and $p$ are functions of the variables $r$ and $z$.

2. There exists $\alpha \in H^{1}\left(\mathcal{D}_{a}\right)$ such that

$$
u_{1}=\alpha(z, r) \cos \theta \text { and } u_{2}=\alpha(z, r) \sin \theta .
$$

Proof. See Section 3.1. 
Let us write $u_{3}=\widetilde{u}_{3}(r, z), p=\widetilde{p}(r, z)$ and introduce $\mathbf{w}=\left(\widetilde{u}_{3}, \alpha\right)=\left(w_{1}, w_{2}\right)$. As a direct consequence of the above proposition, System (2.4) rewrites in cylindrical coordinates

$$
\begin{gathered}
-\mu\left(\Delta \mathbf{w}+\frac{1}{r} \frac{\partial \mathbf{w}}{\partial r}-\frac{1}{r^{2}}\left(\begin{array}{c}
0 \\
w_{2}
\end{array}\right)\right)+\nabla \widetilde{p}=0 \text { in } \mathcal{D}_{a} \\
\nabla \cdot \mathbf{w}+\frac{w_{2}}{r}=0 \text { in } \mathcal{D}_{a} \\
\mathbf{w}(0, r)=\left(u_{0}(r), 0\right) \text { a.e } r \in(0, a(0)) \\
\left(\frac{\partial w_{2}}{\partial z}+\frac{\partial w_{1}}{\partial r}\right)(L, r)=0, \quad\left(-\widetilde{p}+2 \mu \frac{\partial w_{1}}{\partial z}\right)(L, r)=h(r) \text { a.e } r \in(0, a(L)) \\
\frac{\partial w_{1}}{\partial r}(z, 0)=0, w_{2}(z, 0)=0, \mathbf{w}(z, a(z))=0 \text { a.e } z \in(0, L) .
\end{gathered}
$$

The details of this computation are given in the proof of Proposition 2.4, in Section 3.1. Furthermore, the existence of a solution for such a system is guaranteed by the following proposition.

Proposition 2.5. The two dimensional problem (2.9) has a unique solution $\left(\mathbf{w}_{\mathbf{a}}, p_{a}\right)$ in $\left[H^{1}\left(\mathcal{D}_{a}\right)\right]^{2} \times L^{2}\left(\mathcal{D}_{a}\right)$, if $a \in \mathcal{A}_{\infty}$.

Proof. By Theorem 2.1, problem (2.4) has a unique solution $(\mathbf{u}, p)$ in $\left[H^{1}\left(\Omega_{a}\right)\right]^{3} \times L^{2}\left(\Omega_{a}\right)$. Using the result stated in Proposition 2.4, we know that the solution $(\mathbf{u}, p)$ of $(2.4)$ writes $\mathbf{u}=$ $\left(w_{2} \cos \theta, w_{2} \sin \theta, w_{1}\right)$ and $p=\widetilde{p}$ where $(\mathbf{w}, \widetilde{p})$ is solution of $(2.9)$, which ensures the existence of a solution to (2.9). The uniqueness for problem (2.9) follows immediately from the uniqueness of solutions for problem (2.4).

Notice that this system has to be understood in a variational sense, which will make precise in Theorem 2.6.

Using these results, it will be useful to rewrite objective function $J_{0}$ replacing $\mathbf{u}$ by $\left(w_{2} \cos \theta, w_{2} \sin \theta, w_{1}\right)$, where $\mathbf{w}$ is solution of (2.9). If $\Omega=\Omega_{a}$, with $a \in \mathcal{A}_{\infty}$ (in a first time), we denote by $J(a, \mathbf{w})$ the new expression of $J_{0}\left(\Omega_{a}\right)$. A simple but tedious computation (similar to the one of Lemma 3.1) yields

$$
J(a, \mathbf{w})=4 \pi \mu \int_{0}^{L} \int_{0}^{a(z)}\left[\left(\frac{\partial w_{2}}{\partial r}\right)^{2}+\frac{w_{2}^{2}}{r^{2}}+\left(\frac{\partial w_{1}}{\partial z}\right)^{2}+\frac{1}{2}\left(\frac{\partial w_{2}}{\partial z}+\frac{\partial w_{1}}{\partial r}\right)^{2}\right] r \mathrm{~d} r \mathrm{~d} z .
$$

In view of the use of shape optimization techniques, we need to avoid, as much as possible to take into account the regularity constraint on the free boundary. That is why we will state in the next section, an existence result using the variational formulation of (2.9) after extending the solution to a fixed compact set.

REMARK 2. It may be noticed that solving such a Stokes system is equivalent to inverse a fourth order elliptic operator, close to the bilaplacian.

Indeed, since $\mathcal{D}_{a}$ is a two-dimensional domain, one can introduce the so-called stream function $\psi$ (see for instance [33]). Since the divergence-free condition may be rewritten

$$
\frac{\partial}{\partial z}\left(r w_{1}\right)+\frac{\partial}{\partial r}\left(r w_{2}\right)=0 \text { in } \mathcal{D}_{a},
$$

we are led to define $\psi$ by the relations

$$
\begin{cases}r w_{1}=-\frac{\partial \psi}{\partial r} & \text { in } \mathcal{D}_{a} \\ r w_{2}=\frac{\partial \psi}{\partial z} & \text { in } \mathcal{D}_{a} \\ \psi=0 & \text { on } \Gamma_{a}\end{cases}
$$


A tedious computation shows that equations (2.9a)-(2.9b) rewrites in terms of the function $\psi$

$$
\begin{array}{r}
\frac{\partial}{\partial r}\left(\frac{1}{r} \frac{\partial^{2} \psi}{\partial r^{2}}\right)+\frac{\partial^{3} \psi}{\partial z^{2} \partial r}-\frac{1}{r^{3}} \frac{\partial \psi}{\partial r}+\frac{1}{\mu} \frac{\partial}{\partial z}(r \widetilde{p})=0 \text { in } \mathcal{D}_{a} \\
\frac{\partial^{3} \psi}{\partial z^{3}}+\frac{\partial}{\partial r}\left(\frac{1}{r} \frac{\partial^{2} \psi}{\partial r \partial z}\right)-\frac{1}{\mu} \frac{\partial \widetilde{p}}{\partial r}=0 \text { in } \mathcal{D}_{a}
\end{array}
$$

In order to make the pressure term vanish, let us form the equation $\frac{\partial}{\partial r}\left(\frac{1}{r}(2.11)\right)+\frac{\partial}{\partial z}(2.12)$. One gets

$$
\frac{\partial^{4} \psi}{\partial z^{4}}+\frac{1}{r} \frac{\partial^{4} \psi}{\partial r^{4}}+\frac{2}{r} \frac{\partial^{4} \psi}{\partial z^{2} \partial r^{2}}-\left(\frac{2}{r^{2}}+\frac{1}{r^{3}}\right) \frac{\partial^{3} \psi}{\partial r^{3}}-\frac{2}{r^{2}} \frac{\partial \psi^{3}}{\partial r^{2} \partial r}+\frac{2}{r^{3}} \frac{\partial^{2} \psi}{\partial r^{2}}+\frac{4}{r^{5}} \frac{\partial \psi}{\partial r}=0
$$

Moreover, since $\mathbf{w}=0$ on $\Gamma_{a}, \psi$ satisfies

$$
\psi=0 \text { and } \frac{\partial \psi}{\partial n}=0 \text { on } \Gamma_{a}
$$

2.4. Main result: shape existence in the class of cylindrical domains. In Subsection 2.2, a general shape existence result for three dimensional domains has been stated. Unfortunately, this result is hardly workable, since the boundary of the quasi open set $\Omega$ may be very irregular (roughly speaking, when the boundary of the domain is locally represented by the graph of a function which regularity is less than $W^{1, p}, p \geq 2$ ). In such a case, it is sometimes possible to define a solution of the Stokes or Navier-Stokes system, as it is emphasized in [7, 8, 24, 25, 32].

This section is devoted to the a shape existence results for domains enjoying a cylindrical symmetry property. We do not assume any longer that the free boundary is $W^{1, \infty}$. Let $h_{0}: \mathbb{R} \rightarrow \mathbb{R}$ and $h_{L}: \mathbb{R} \rightarrow \mathbb{R}$ be two given continuous functions.

Let us introduce the class of admissible domains $\mathbb{U}_{p}$, for $p \geq 2$ by

$$
\begin{aligned}
\mathbb{U}_{p} & =\left\{\mathcal{D}_{a}=\{(z, a(z)) \mid 0 \leq z \leq L\} \mid a \in \mathcal{U}_{p}\right\} \\
\text { where } \mathcal{U}_{p} & =\left\{a \in \mathcal{A}_{p} \mid\left\|a^{\prime}\right\|_{L^{p}} \leq M, h_{0}(a(0))=h_{L}(a(L))=0\right\}, \\
\text { and } \mathcal{A}_{p} & =\left\{a \in W^{1, p}(0, L) \mid a_{0} \leq a(z) \leq a_{1} \text { for } z \in(0, L)\right\},
\end{aligned}
$$

where $h_{0}$ and $h_{L}$ are chosen to be compatible with the pointwise constraint satisfied by each element of $\mathcal{A}_{p}$.

It may be noticed that the compact embedding $W^{1, p}(0, L) \hookrightarrow \mathcal{C}^{0}([0, L])$ for the standard $L^{\infty}$. topology yields in particular the existence of $a_{M}>a_{0}$ such that $\|a\|_{\infty} \leq a_{M}$ for any $a \in \mathbb{U}_{p}$. In what follows, we will make the assumption that

$$
\exists \eta>0 \mid[0, \eta] \subset \operatorname{supp}\left(u_{0}\right)
$$

We will see in the sequel that it permits to neglect the pointwise constraint $a \geq a_{0}$ almost everywhere.

For a given $p \geq 2$, we consider the shape optimization problem

$$
\left\{\begin{array}{l}
\min J_{0}(\Omega) \\
\Omega \in \mathcal{O}_{\mathrm{cyl}}^{p}=\left\{(r \cos \theta, r \sin \theta, z), \exists a \in \mathcal{U}_{p}, 0 \leq r<a(z), \theta \in \mathbb{T}, 0<z<L\right\},
\end{array}\right.
$$

where, the functional $J_{0}(\Omega)$ denotes the dissipated energy, defined, in the case of a sufficiently regular domain $\Omega$, for instance with Lipschitz boundary, by equation (2.5). Nevertheless, in the 
case where the boundary is not regular enough, we can define $J_{0}(\Omega)$ using Lemma 2.7 by

$$
J_{0}(\Omega)=2 \mu \inf _{\substack{\mathbf{u} \in\left[H^{1}(\Omega)\right]^{3}, \nabla \cdot \mathbf{u}=0 \\ \mathbf{u}=\mathbf{u}_{0} \text { on } E \\ \mathbf{u}=0 \text { on } \Sigma}} \int_{\Omega}|\varepsilon(\mathbf{u})|^{2} d x .
$$

An other way to define $J_{0}(\Omega)$ consists in defining $\mathbf{u}_{\Omega}$ thanks to a weak formulation, well adapted for the class of axisymmetric domains with respect to the $(O z)$-axis. In particular, since any domain $\Omega \in \mathcal{O}_{\text {cyl }}^{p}$ is contained in the fixed compact set

$$
\mathcal{D}=\left\{(r \cos \theta, r \sin \theta, z), r \in\left[0, a_{M}\right], \theta \in \mathbb{T}, z \in[0, L]\right\},
$$

we have the temptation to write a weak formulation of the Stokes system on $\mathcal{D}$ which lateral boundary is quite regular.

For that purpose, let us define, the Sobolev spaces

$$
\begin{aligned}
H_{d}\left(\mathcal{D}_{a}\right) & =\left\{\varphi=\left(\varphi_{1}, \varphi_{2}\right) \in\left[H^{1}\left(\mathcal{D}_{a}\right)\right]^{2} \mid \nabla \cdot \varphi+\frac{\varphi_{2}}{r}=0 \text { on } \mathcal{D}_{a}\right\}, \\
H_{d, 0}\left(\mathcal{D}_{a}\right) & =\left\{\varphi=\left(\varphi_{1}, \varphi_{2}\right) \in\left[H^{1}\left(\mathcal{D}_{a}\right)\right]^{2} \mid \nabla \cdot \varphi+\frac{\varphi_{2}}{r}=0 \text { on } \mathcal{D}_{a} \text { and } \varphi=0 \text { on } \Gamma_{0} \cup \Gamma_{a}\right\} .
\end{aligned}
$$

The following result plays a crucial role in the proof of the existence of solutions for the shape optimization problem (2.17).

TheOREM 2.6. Let $p \geq 2$ and $\Omega \in \mathcal{O}_{\text {cyl }}^{p}$, associated with a two-dimensional domain $\mathcal{D}_{a}$ in cylindrical coordinates.

1. Let $a \in \mathcal{A}_{p}$. If $\mathbf{w}_{\mathbf{a}} \in H_{d}\left(\mathcal{D}_{a}\right)$ is solution of (2.9), then, the function $\mathbf{w}$ defined by

$$
\mathbf{w}= \begin{cases}\mathbf{w}_{\mathbf{a}} & \text { in } \mathcal{D}_{a} \\ 0 & \text { in } \mathcal{D} \backslash \mathcal{D}_{a}\end{cases}
$$

satisfies

$$
\forall \mathbf{z} \in H_{d}\left(\mathcal{D}_{a}\right) \cap \mathcal{C}_{c}^{\infty}\left(\overline{\mathcal{D}_{a}} \backslash \Gamma_{a}\right), 2 \mu \int_{\mathcal{D}}\left(\varepsilon_{2}(\mathbf{w}): \varepsilon_{2}(\mathbf{z})+\frac{w_{2} z_{2}}{r^{2}}\right) r \mathrm{~d} r \mathrm{~d} z=0 .
$$

and $\mathbf{w}=0$ on $\Gamma_{a}, \mathbf{w}=\left(u_{0}, 0\right)$ on $\Gamma_{0}$.

2. Conversely, let $\mathbf{w} \in\left[H^{1}(\mathcal{D})\right]^{2}$. If there exist $a \in \mathcal{A}_{p}$ and $\mathbf{w}_{\mathbf{a}}$ such that

$$
\mathbf{w}= \begin{cases}\mathbf{w}_{\mathbf{a}} & \text { in } \mathcal{D}_{a} \\ 0 & \text { in } \mathcal{D} \backslash \mathcal{D}_{a}\end{cases}
$$

and if $\mathbf{w}$ satisfies (2.19) for any $\varphi \in H_{d}\left(\mathcal{D}_{a}\right) \cap \mathcal{C}_{c}^{\infty}\left(\overline{\mathcal{D}_{a}} \backslash \Gamma_{a}\right)$, then $\mathbf{w}_{\mathbf{a}}$ is solution of (2.9).

\section{Proof. See Section 3.2.}

The result stated in Theorem 2.6 combined with the results of Section 2.3, (in particular Proposition 2.4) drives us to consider a new shape optimization problem, over axisymmetric domains, directly deduced from the initial general shape optimization problem (2.17). Indeed, let us introduce the problem

$$
\left\{\begin{array}{l}
\min J\left(a, \mathbf{w}_{\mathbf{a}}\right) \\
\mathbf{w}_{\mathbf{a}} \text { is solution of }(2.19) \\
a \in \mathcal{U}_{p}
\end{array}\right.
$$




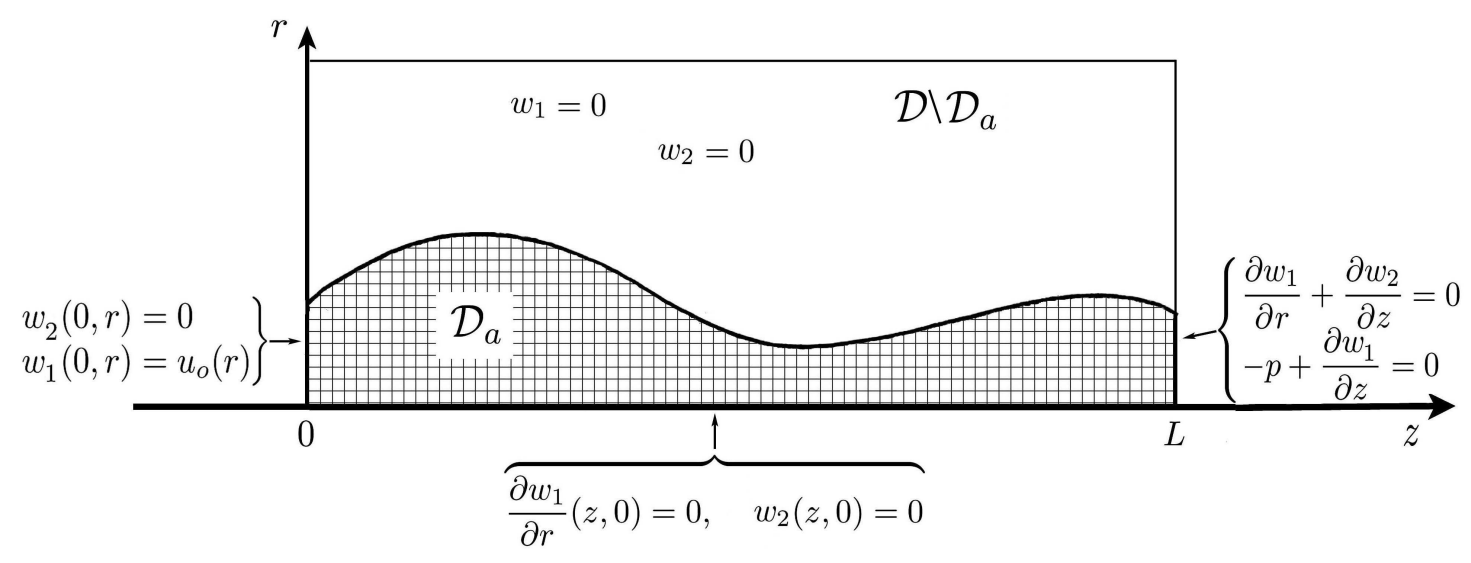

FIG. 2.2. Domains $\mathcal{D}$ and $\mathcal{D}_{a}$ with boundary conditions

where $J(a, \mathbf{w})$ is defined by $(2.10)$.

In fact, keeping in mind that it seems better to write all the integrals on the fixed compact set $\mathcal{D}$, it is possible to extend $\mathbf{w}_{\mathbf{a}}$ by 0 , so that the shape optimization problem becomes

$$
\left\{\begin{array}{l}
\min J\left(\mathbf{w}_{\mathbf{a}}\right) \\
\mathbf{w}_{\mathbf{a}} \text { is solution of }(2.19) \\
a \in \mathcal{U}_{p}
\end{array}\right.
$$

where

$$
J(\mathbf{w})=4 \pi \mu \int_{\mathcal{D}}\left[\left(\frac{\partial w_{2}}{\partial r}\right)^{2}+\frac{w_{2}^{2}}{r^{2}}+\left(\frac{\partial w_{1}}{\partial z}\right)^{2}+\frac{1}{2}\left(\frac{\partial w_{2}}{\partial z}+\frac{\partial w_{1}}{\partial r}\right)^{2}\right] r \mathrm{~d} r \mathrm{~d} z .
$$

The following theorem constitutes the main result of this section.

Theorem 2.7. Let $p \geq 2$. Problem (2.21) has (at least) a solution.

Proof. See Section 3.3. प

Note that, in general, we do not have uniqueness of the minimizer in shape optimization (see e.g. $[1,14])$.

Furthermore, for the needs of future numerical computations of the optimal shape, one may neglect the pointwise constraint $a \geq a_{0}$ and replace it by $a \geq 0$ for $z \in(0, L)$. Indeed, the result stated in Theorem 2.7 is a bit more general since it may be noticed that, because of the "divergence-free" constraint, one has for $\bar{z} \in(0, L)$ fixed,

$$
\int_{\operatorname{supp}\left(u_{0}\right)} u_{0}(r) r \mathrm{~d} r=\int_{0}^{a(\bar{z})} w_{1}(r, \bar{z}) r \mathrm{~d} r
$$

where $\operatorname{supp}\left(u_{0}\right)$ denotes the support of the data $u_{0}$ that is supposed of strictly positive measure. This identity comes directly from the integration of the "divergence-free" condition on the restriction of the domain $\Omega_{a}$ between the hyperplane $z=0$ and $z=\bar{z}$. Thus, the optimal graph $a$ cannot vanish because else, the above identity would not be guaranteed.

\section{Proofs.}


3.1. Proof of Proposition 2.4. It is assumed that $u_{0}$ only depends on $r$. The pair $(\mathbf{u}, p)$ denotes the unique solution of System (2.4). Let $\mathcal{L}$ be the operator

$$
\mathcal{L}=x_{2} \frac{\partial}{\partial x_{1}}-x_{1} \frac{\partial}{\partial x_{2}}
$$

the partial derivatives being understood in the sense of distributions. This operator stands actually for the differentiation with respect to the polar angle $\theta$, applied to a function expressed in cylindrical coordinates. We easily check that

$$
\mathcal{L}\left(\frac{\partial v}{\partial x_{1}}\right)=\frac{\partial \mathcal{L}(v)}{\partial x_{1}}+\frac{\partial v}{\partial x_{2}}, \mathcal{L}\left(\frac{\partial v}{\partial x_{2}}\right)=\frac{\partial \mathcal{L}(v)}{\partial x_{2}}-\frac{\partial v}{\partial x_{1}} \text { and } \mathcal{L}\left(\frac{\partial v}{\partial x_{3}}\right)=\frac{\partial \mathcal{L}(v)}{\partial x_{3}} .
$$

Let us introduce $\widehat{\mathbf{u}}=\mathcal{L}(\mathbf{u})=\left(\widehat{u}_{1}, \widehat{u}_{2}, \widehat{u}_{3}\right)$ and $\widehat{p}=\mathcal{L}(p)$. By applying $\mathcal{L}$ to System (2.4), we get

$$
\left\{\begin{aligned}
-\mu \Delta \widehat{u}_{1}+\frac{\partial \widehat{p}}{\partial x_{1}}+\frac{\partial p}{\partial x_{2}}=0 & \text { in } \Omega_{a} \\
-\mu \Delta \widehat{u}_{2}+\frac{\partial \hat{p}}{\partial x_{2}}-\frac{\partial p}{\partial x_{1}}=0 & \text { in } \Omega_{a} \\
-\mu \Delta \widehat{u}_{3}+\frac{\partial \widehat{p}}{\partial x_{3}}=0 & \text { in } \Omega_{a} \\
\nabla \cdot \widehat{\mathbf{u}}+\frac{\partial u_{1}}{\partial x_{2}}-\frac{\partial u_{2}}{\partial x_{1}}=0 & \text { in } \Omega_{a} \\
\sigma(\widehat{\mathbf{u}}, \widehat{p}) \cdot \mathbf{n}=\left(\nabla u_{3}\right)^{\perp} & \text { on } \Sigma_{a} \cup E_{a}
\end{aligned}\right.
$$

where $\left(\nabla u_{3}\right)^{\perp}=\left(-\frac{\partial u_{3}}{\partial x_{2}}, \frac{\partial u_{3}}{\partial x_{1}}, 0\right)^{\top}$. Notice that the initial hypothesis that $u_{0}$ is a function of the variable $r$ has been used to obtain the homogeneous Dirichlet boundary condition for $\widehat{\mathbf{u}}$ on $\Sigma_{a} \cup E_{a}$.

Let us now introduce the following new functions:

$$
v_{1}=\widehat{u}_{1}-u_{2}, v_{2}=\widehat{u}_{2}+u_{1}, \text { and } v_{3}=\widehat{u}_{3} .
$$

Then, System (3.1) rewrites in terms of $v_{1}, v_{2}, v_{3}$ and $\widehat{p}$,

$$
\left\{\begin{aligned}
-\mu \Delta \mathbf{v}+\nabla \widehat{p}=0 & \text { in } \Omega_{a} \\
\nabla \cdot \mathbf{v}=0 & \text { in } \Omega_{a} \\
\mathbf{v}=0 & \text { on } \Sigma_{a} \cup E_{a} \\
\sigma(\mathbf{v}, \widehat{p}) \cdot \mathbf{n}=0 & \text { on } S_{a}
\end{aligned}\right.
$$

It is well known (see e.g. $[3,13,33]$ ) that this system has a unique solution, therefore,

$$
v_{1}=v_{2}=v_{3}=\widehat{p} \equiv 0 .
$$

The fact that $\mathcal{L}\left(u_{3}\right)$ and $\mathcal{L}(p)$ vanish proves the first point of Proposition 2.4. We deduce the existence of $\widetilde{u}_{3}$ and $\widetilde{p}$ such that

$$
p\left(x_{1}, x_{2}, x_{3}\right)=\widetilde{p}(z, r) \text { and } u_{3}\left(x_{1}, x_{2}, x_{3}\right)=\widetilde{u}_{3}(z, r) \text { for }\left(x_{1}, x_{2}, x_{3}\right) \in \Omega_{a},
$$

where $(z, r)$ has been defined by (2.8). Furthermore, it has been proved that $\mathcal{L}\left(u_{1}\right)=u_{2}$ and $\mathcal{L}\left(u_{2}\right)=-u_{1}$. Therefore, applying once more the operator $\mathcal{L}$ yields $\mathcal{L} \circ \mathcal{L}\left(u_{1}\right)+u_{1}=0$ and then, there exist two functions $\alpha$ and $\beta$ of the variables $r$ and $z$, in the space $H^{1}\left(\mathcal{D}_{a}\right)$ such that

$$
u_{1}=\alpha(z, r) \cos \theta+\beta(z, r) \sin \theta \text { and } u_{2}=\alpha(z, r) \sin \theta-\beta(z, r) \cos \theta .
$$


To end the proof, it remains to prove that the function $\beta$ vanishes identically. For that purpose, let us write down the partial differential equations satisfied by $\alpha$ and $\beta$. Using standard change of variable formula, we get for almost every $\theta \in(0,2 \pi)$ and $(z, r) \in(0, a(z)) \times(0, L)$,

$$
\left\{\begin{array}{l}
-\mu\left(\frac{\partial^{2} \alpha}{\partial r^{2}}+\frac{1}{r} \frac{\partial \alpha}{\partial r}-\frac{\alpha}{r^{2}}+\frac{\partial^{2} \alpha}{\partial z^{2}}\right) \cos \theta+\frac{\partial \widetilde{p}}{\partial r} \cos \theta-\mu\left(\frac{\partial^{2} \beta}{\partial r^{2}}+\frac{1}{r} \frac{\partial \beta}{\partial r}-\frac{\beta}{r^{2}}+\frac{\partial^{2} \beta}{\partial z^{2}}\right) \sin \theta=0 \\
-\mu\left(\frac{\partial^{2} \alpha}{\partial r^{2}}+\frac{1}{r} \frac{\partial \alpha}{\partial r}-\frac{\alpha}{r^{2}}+\frac{\partial^{2} \alpha}{\partial z^{2}}\right) \sin \theta+\frac{\partial \widetilde{p}}{\partial r} \sin \theta+\mu\left(\frac{\partial^{2} \beta}{\partial r^{2}}+\frac{1}{r} \frac{\partial \beta}{\partial r}-\frac{\beta}{r^{2}}+\frac{\partial^{2} \beta}{\partial z^{2}}\right) \sin \theta=0
\end{array}\right.
$$

which formally yields

$$
\begin{array}{r}
-\mu\left(\frac{\partial^{2} \alpha}{\partial r^{2}}+\frac{1}{r} \frac{\partial \alpha}{\partial r}-\frac{\alpha}{r^{2}}+\frac{\partial^{2} \alpha}{\partial z^{2}}\right)+\frac{\partial \widetilde{p}}{\partial r}=0 \text { in } \mathcal{D}_{a} \\
\frac{\partial^{2} \beta}{\partial r^{2}}+\frac{1}{r} \frac{\partial \beta}{\partial r}-\frac{\beta}{r^{2}}+\frac{\partial^{2} \beta}{\partial z^{2}}=0 \text { in } \mathcal{D}_{a}
\end{array}
$$

The divergence condition rewrites

$$
\frac{\alpha}{r}+\frac{\partial \alpha}{\partial r}+\frac{\partial \widetilde{u}_{3}}{\partial z}=0 \text { in } \mathcal{D}_{a}
$$

Now, let us precise the boundary conditions on $\alpha$ and $\beta$.

- $\mathbf{u}=0$ on $\Sigma_{a}$ provides

$$
\alpha(z, a(z))=\beta(z, a(z))=\widetilde{u}_{3}(z, a(z))=0, \text { for } z \in(0, L) .
$$

- $\mathbf{u}=\left(0,0, u_{0}\right)$ on $E_{a}$ provides

$$
\alpha(0, r)=\beta(0, r)=0, \widetilde{u}_{3}(0, r)=u_{0}(r), \text { for } r \in(0, a(0)) .
$$

- $\sigma(\mathbf{u}, p) \cdot \mathbf{n}=0$ on $S_{a}$ provides

$$
\frac{\partial \alpha}{\partial z}(L, r)+\frac{\partial \widetilde{u}_{3}}{\partial r}(L, r)=\frac{\partial \beta}{\partial z}(L, r)=0,-\widetilde{p}+2 \mu \frac{\partial \widetilde{u}_{3}}{\partial z}(L, r)=0, \text { for } r \in(0, a(L)) .
$$

- Furthermore, in order to obtain a well-posed system on $\alpha$ and $\beta$, we have to add a transmission boundary condition, directly coming from the symmetry property proved previously, without any additional regularity assumption on $\mathbf{u}$, that is

$$
\frac{\partial \widetilde{u}_{3}}{\partial r}(z, 0)=0, \text { for } z \in(0, L)
$$

obtained by writing that for almost every $x_{3} \in(0, L),\left(x_{1}, x_{2}\right)$ such that $x_{1}^{2}+x_{2}^{2} \leq \epsilon, \epsilon>0$, one has $u_{3}\left(x_{1}, x_{2}, x_{3}\right)=u_{3}\left(-x_{1}, x_{2}, x_{3}\right)$, and making an Taylor expansion with respect to $x_{1}$ and $x_{2}$ at the first order. Similarly,

$$
\frac{\partial \alpha}{\partial r}(z, 0)=\frac{\partial \beta}{\partial r}(z, 0)=0, \text { for } z \in(0, L),
$$

Moreover, using the divergence condition (3.5), we get, by making $r$ tend to zero,

$$
\alpha(z, 0)=0, \text { for } z \in(0, L)
$$


Now, let us prove that $\beta \equiv 0$. Let us recall that, according to the previous analysis, $\beta$ is solution of

$$
\left\{\begin{array}{l}
\Delta \beta+\frac{1}{r} \frac{\partial \beta}{\partial r}-\frac{\beta}{r^{2}}=0 \text { in } \mathcal{D}_{a} \\
\beta(z, a(z))=0, \text { for } z \in(0, L), \beta(0, r)=0 \text { for } r \in(0, a(0)), \\
\frac{\partial \beta}{\partial z}(L, r)=0 \text { for } r \in(0, a(L)), \frac{\partial \beta}{\partial r}(z, 0)=0 \text { for } z \in(0, L) .
\end{array}\right.
$$

We use $r^{2} \beta \in H^{1}\left(\mathcal{D}_{a}\right)$ as a test function to perform an integration by parts. Indeed, the first equation of the above system has to be understood in the variational sense. We get

$$
\begin{aligned}
0 & =-\int_{\mathcal{D}_{a}} r^{2}|\nabla \beta|^{2} \mathrm{~d} r \mathrm{~d} z-\int_{\mathcal{D}_{a}} r \beta \frac{\partial \beta}{\partial r} \mathrm{~d} r \mathrm{~d} z-\int_{\mathcal{D}_{a}} \beta^{2} \mathrm{~d} r \mathrm{~d} z \\
& =-\int_{\mathcal{D}_{a}} r^{2}|\nabla \beta|^{2} \mathrm{~d} r \mathrm{~d} z-\frac{1}{2} \int_{\mathcal{D}_{a}} \beta^{2} \mathrm{~d} r \mathrm{~d} z .
\end{aligned}
$$

Hence,

$$
\beta=\frac{\partial \beta}{\partial z}=\frac{\partial \beta}{\partial r} \equiv 0
$$

Finally, the solution $(\mathbf{u}, p)$ of System (2.4) satisfies

$$
u_{1}=\alpha(z, r) \cos \theta, u_{2}=\alpha(z, r) \sin \theta, u_{3}=\widetilde{u}_{3}(z, r), p=\widetilde{p}(z, r),
$$

and according to the previous analysis, by setting $\mathbf{w}=\left(\widetilde{u}_{3}, \alpha\right)=\left(w_{1}, w_{2}\right)$, we prove moreover that $\mathbf{w}$ is solution of the two dimensional system (2.9).

3.2. Proof of Theorem 2.6. Let us begin with an integration by parts formula, adapted for the special case of the Stokes operator defined on domains having a cylindrical symmetry.

LEMma 3.1. Let $a \in W^{1, p}(0, L)$, with $p \geq 2$, and $\mathbf{w}$ be a $H^{2}$ vector field defined on the domain $\mathcal{D}_{a}$. Let us assume that $\mathbf{w}$ satisfies the "divergence-free condition for cylindrical domains", that is

$$
\frac{\partial w_{2}}{\partial z}+\frac{1}{r} \frac{\partial}{\partial r}\left(r w_{1}\right)=0 \text { for }(r, z) \in \mathcal{D}_{a} .
$$

Then for any $\mathbf{z} \in\left[H^{1}\left(\mathcal{D}_{a}\right)\right]^{2}$, one has

$$
2 \int_{\mathcal{D}_{a}}\left(\varepsilon_{2}(\mathbf{w}): \varepsilon_{2}(\mathbf{z})+\frac{w_{2} z_{2}}{r^{2}}\right) r \mathrm{~d} r \mathrm{~d} z=-\int_{\mathcal{D}_{a}} L \mathbf{w} \cdot \mathbf{z} r \mathrm{~d} r \mathrm{~d} z+2 \int_{\partial \mathcal{D}_{a}} \varepsilon_{2}(\mathbf{w}) \cdot \mathbf{n} \cdot \mathbf{z d} \sigma
$$

where

- $\varepsilon_{2}(\mathbf{w})=\frac{1}{2}\left(\nabla \mathbf{w}+[\nabla \mathbf{w}]^{\top}\right)$, and $\nabla \mathbf{w}$ denotes the Jacobian matrix with respect to the variables $(z, r)$;

- $L=\Delta+\frac{\partial}{\partial r}-\frac{1}{r^{2}}\left(\begin{array}{ll}0 & 0 \\ 0 & 1\end{array}\right) I$;

- $\mathrm{d} \sigma$ is the surface measure associated with the measure $r \mathrm{~d} r \mathrm{~d} z$.

Proof. A direct way may be used to prove this identity. Nevertheless, we decided here to use the well known identity (see e.g. [3])

$$
\forall(\mathbf{u}, \mathbf{v}) \in\left[H^{2}(\Omega)\right]^{2}, \int_{\Omega} \varepsilon(\mathbf{u}): \varepsilon(\mathbf{v}) \mathrm{d} x=-\int_{\Omega}(\Delta \mathbf{u}+\nabla(\nabla \cdot \mathbf{u})) \cdot \mathbf{v} \mathrm{d} x+\int_{\partial \Omega} \varepsilon(\mathbf{u}) \cdot \mathbf{n} \cdot \mathbf{v} \mathrm{d} s,
$$


where $\Omega$ is a bounded regular (for instance Lipschitz) domain of $\mathbb{R}^{3}$. We will rewrite this formula in the particular case where $\Omega=\Omega_{a}$ and there exist $\left(\alpha, \widetilde{u}_{3}\right)$ and $\left(\gamma, \widetilde{v}_{3}\right)$ in $\left[H^{2}\left(\mathcal{D}_{a}\right)\right]^{2}$ such that

$$
\mathbf{u}=\left(\alpha(r, z) \cos \theta, \alpha(r, z) \sin \theta, \widetilde{u}_{3}\right)^{\top} \text { and } \mathbf{v}=\left(\gamma(r, z) \cos \theta, \gamma(r, z) \sin \theta, \widetilde{v}_{3}\right)^{\top} .
$$

Nevertheless, we have to pay a bit attention to the regularity of the boundary of $\Omega_{a}$, and more precisely to $\Sigma_{a}$. Indeed, since $a \in W^{1, p}(0, L)$ with $p \geq 2, a$ is a priori not Lipschitz. The only restriction on the function $a$ for writing the above formula lies in the fact that we must define the normal vector $\mathbf{n}$ almost everywhere on the boundary $\Sigma_{a}$ and the boundary integrals must exist. In fact, this formula remains true in our case. Indeed, one easily checks

$$
\mathbf{n}=\frac{1}{\sqrt{1+a^{\prime 2}(z)}}\left(\begin{array}{c}
\cos \theta \\
\sin \theta \\
-a^{\prime}(z)
\end{array}\right) \text { on } \Sigma_{a} .
$$

Now, $z \mapsto \frac{1}{\sqrt{1+a^{\prime 2}(z)}}$ belongs to $L^{\infty}(0, L)$ while $z \mapsto a^{\prime}(z)$ belongs to $L^{2}(0, L)$, since $a \in W^{1, p}(0, L)$ with $p \geq 2$. Therefore, $\mathbf{n}$ is defined almost everywhere on $\Sigma_{a}$ and belongs to $L^{2}\left(\Sigma_{a}\right)$. Hence, the boundary integral in the previous integration by part formula is well defined, as a product of three functions in $L^{2}\left(\Sigma_{a}\right)$, thanks to the usual trace theorems. Easy, but lengthly computations prove that

$$
\begin{aligned}
\int_{\Omega_{a}} \varepsilon(\mathbf{u}): \varepsilon(\mathbf{v}) \mathrm{d} x & =\pi \int_{\mathcal{D}_{a}}\left[2\left(\frac{\partial \alpha}{\partial r} \frac{\partial \gamma}{\partial r}+\frac{1}{r^{2}} \alpha \gamma+\frac{\partial \widetilde{u}_{3}}{\partial z} \frac{\partial \widetilde{v}_{3}}{\partial z}\right)+\left(\frac{\partial \alpha}{\partial z}+\frac{\partial \widetilde{u}_{3}}{\partial r}\right)\left(\frac{\partial \gamma}{\partial z}+\frac{\partial \widetilde{v}_{3}}{\partial r}\right)\right] r \mathrm{~d} r \mathrm{~d} z \\
& =2 \pi \int_{\mathcal{D}_{a}}\left(\varepsilon_{2}(\mathbf{w}): \varepsilon_{2}(\mathbf{z})+\frac{1}{r^{2}} \alpha \gamma\right) r \mathrm{~d} r \mathrm{~d} z
\end{aligned}
$$

where $\mathbf{w}=\left(\widetilde{u}_{3}, \alpha\right)^{\top}$ and $\mathbf{z}=\left(\widetilde{v}_{3}, \gamma\right)^{\top}$. Using the new notation we get

$$
\int_{\Omega_{a}} \varepsilon(\mathbf{u}): \varepsilon(\mathbf{v}) \mathrm{d} x=2 \pi \int_{\mathcal{D}_{a}}\left(\varepsilon_{2}(\mathbf{w}): \varepsilon_{2}(\mathbf{z})+\frac{1}{r^{2}} w_{2} z_{2}\right) r \mathrm{~d} r \mathrm{~d} z .
$$

Similarly, using the well known expression of the Laplace operator in polar coordinates permits to recover easily the right hand term in equation (3.8).

Now, let us make the boundary integrals precise. Noticing that $\mathbf{n}=\left(n_{r} \cos \theta, n_{r} \sin \theta, n_{z}\right)$, we show that

$$
\varepsilon(\mathbf{u}) \cdot \mathbf{n}=\left(\begin{array}{c}
\cos \theta \frac{\partial \alpha}{\partial r} n_{r}+\frac{1}{2} \cos \theta\left(\frac{\partial \alpha}{\partial z}+\frac{\partial \widetilde{u}_{3}}{\partial r}\right) n_{z} \\
\sin \theta \frac{\partial \alpha}{\partial r} n_{r}+\frac{1}{2} \sin \theta\left(\frac{\partial \alpha}{\partial z}+\frac{\partial \widetilde{u}_{3}}{\partial r}\right) n_{z} \\
\left(\frac{\partial \alpha}{\partial z}+\frac{\partial \widetilde{u}_{3}}{\partial r}\right) n_{r}+\frac{\partial \widetilde{u}_{3}}{\partial z} n_{z}
\end{array}\right)
$$

driving easily to the boundary expression in the right hand side of (3.8).

The following proposition is the key point of the proof of Theorem 2.6.

Proposition 3.2. Let $\mathbf{w} \in H_{d}\left(\mathcal{D}_{a}\right)$ be solution of problem (2.9). Then, one has

$$
\forall \mathbf{z} \in H_{d, 0}\left(\mathcal{D}_{a}\right), 2 \mu \int_{\mathcal{D}_{a}}\left(\varepsilon_{2}(\mathbf{w}): \varepsilon_{2}(\mathbf{z})+\frac{w_{2} z_{2}}{r^{2}}\right) r \mathrm{~d} r \mathrm{~d} z=0 .
$$

Conversely, if $\mathbf{w} \in H_{d}\left(\mathcal{D}_{a}\right)$ satisfies Relation (3.11) and if $\mathbf{w}=0$ on $\Gamma_{a}$ and $\mathbf{w}=\left(u_{0}, 0\right)$ on $\Gamma_{0}$, then $\mathbf{w}$ is the unique solution of problem (2.9). 
Proof. Let us formally multiply the first equation of (2.9) by $\mathbf{z}$ and then integrate by parts, using the identity stated in Lemma 3.1. We obtain

$$
\begin{gathered}
2 \mu \int_{\mathcal{D}_{a}}\left(\varepsilon_{2}(\mathbf{w}): \varepsilon_{2}(\mathbf{z})+\frac{w_{2} z_{2}}{r^{2}}\right) r \mathrm{~d} r \mathrm{~d} z \\
+2 \mu \int_{\partial \mathcal{D}_{a}} \varepsilon_{2}(\mathbf{w}) \cdot \mathbf{n} \cdot \mathbf{z}-\left.\int_{0}^{a(L)} \widetilde{p} z_{1}\right|_{z=L} r \mathrm{~d} r+\left.\int_{0}^{a(0)} \tilde{p} z_{1}\right|_{z=L} r \mathrm{~d} r=0,
\end{gathered}
$$

which proves that (3.11) is satisfied. The converse sense is immediate.

We are now in position to end the proof of Theorem 2.6. It remains to extend the variational formulation (3.11) to $\mathcal{D}$ and to prove the well-posed character of this problem.

For any function $\mathbf{w}_{\mathbf{a}}$ defined on $\mathcal{D}_{a}$, we denote by $\widetilde{\mathbf{w}}_{\mathbf{a}}$ the extension of $\mathbf{w}_{\mathbf{a}}$ by 0 in $\mathcal{D}$, that is

$$
\widetilde{\mathbf{w}}_{\mathbf{a}}= \begin{cases}\mathbf{w}_{\mathbf{a}} & \text { on } \mathcal{D}_{a} \\ 0 & \text { on } \mathcal{D} \backslash \mathcal{D}_{a} .\end{cases}
$$

Let $\mathcal{A}$ be the bilinear form defined on $\left[H_{d}(\mathcal{D})\right]^{2}$ by

$$
\mathcal{A}(\mathbf{w}, \mathbf{z})=2 \mu \int_{\mathcal{D}}\left(\varepsilon_{2}(\mathbf{w}): \varepsilon_{2}(\mathbf{z})+\frac{w_{2} z_{2}}{r^{2}}\right) r \mathrm{~d} r \mathrm{~d} z
$$

and $\mathcal{L}$ the identically zero linear form defined on $H_{d}(\mathcal{D})$, so that we are interested in the solution of

$$
\mathcal{A}(\mathbf{w}, \mathbf{z})=\mathcal{L}(\mathbf{z})=0,
$$

where $\mathbf{w}$ and $\mathbf{z}$ are chosen as in the statement of Theorem 2.6.

Existence and uniqueness of a solution for the variational formulation (2.19) come from a direct application of Lax-Milgram's Theorem, using Korn's inequality on $\mathcal{D}$ to get the ellipticity of $\mathcal{A}$ (see e.g. [15]).

3.3. Proof of Theorem 2.7. Let $\left(a_{n}\right)_{n \in \mathbb{N}}$ be a minimizing sequence. $\left(a_{n}\right)_{n \in \mathbb{N}}$ is bounded in $W^{1, p}(0, L)$ and then, converges weakly in $W^{1, p}(0, L)$, strongly in $L^{\infty}(0, L)$ to $a \in \mathcal{U}_{p}$, because of the compactness of the embedding $W^{1, p}(0, L) \hookrightarrow L^{\infty}(0, L)$, for $p>1$, and of the fact that $h_{0}$ and $h_{L}$ are continuous real functions. Let us denote by $\mathbf{w}_{\mathbf{n}}$ the weak solution of problem (2.9) in $\mathcal{D}_{n}=\mathcal{D}_{a_{n}}$. The convergence holds up to a subsequence that we denote similarly in the sequel, with a slight abuse of notations. Now, let us recall that, because of Korn's inequality applied on $\mathcal{D}$, the norms $H^{1}, H_{0}^{1}$ and $\|\cdot\|_{\varepsilon}$ are equivalent (see for instance $[3,15]$ ). Therefore $J$ is coercive. As $\left(J\left(\mathbf{w}_{\mathbf{n}}\right)\right)_{n \in \mathbb{N}}$ is bounded $\left(\left(\mathbf{w}_{\mathbf{n}}\right)_{n \in \mathbb{N}}\right.$ is a minimizing sequence), then the sequence $\left(\mathbf{w}_{\mathbf{n}}\right)_{n \in \mathbb{N}}$ is bounded in $H^{1}(\mathcal{D})$ and then converges weakly to some $\widetilde{\mathbf{w}}$ in $H^{1}(\mathcal{D})$ up to a subsequence. The whole problem consists in showing that $\widetilde{\mathbf{w}}$ vanishes on $\mathcal{D} \backslash \mathcal{D}_{a}$ and that its restriction on $\mathcal{D}_{a}$ is solution of $(2.9)$ in $\mathcal{D}_{a}$.

Let us show that $\widetilde{\mathbf{w}} \equiv 0$ on $\mathcal{D} \backslash \mathcal{D}_{a}$. Let $\epsilon>0$. As a consequence of the uniform convergence of $a_{n}$ to $a$, one can find $n_{\epsilon}>0$ such that

$$
\forall n \geq n_{\epsilon}, \forall z \in[0, L], a_{n}(z) \leq a(z)+\epsilon .
$$

Let $z \in[0, L]$ and $r \in\left[a(z)+\epsilon, a_{M}\right]$. For any $n \geq n_{\epsilon},(z, r) \in \mathcal{D} \backslash \mathcal{D}_{n}$ and then, $\mathbf{w}_{\mathbf{n}}(z, r)=0$. Since $\mathbf{w}_{\mathbf{n}}(z, r)$ converges to $\widetilde{\mathbf{w}}(z, r)$ almost everywhere, we have

$$
\forall \epsilon>0, \forall z \in[0, L], \forall r \in\left[a(z)+\epsilon, a_{M}\right], \widetilde{\mathbf{w}}(z, r)=0 .
$$


Making $\epsilon$ tend to 0 yields $\widetilde{\mathbf{w}} \equiv 0$ on $\overline{\mathcal{D} \backslash \mathcal{D}_{a}}$. Since the divergence condition is preserved, it is easy to see that $\mathbf{w}=\widetilde{\mathbf{w}}_{\mid \mathcal{D}_{a}}$ belongs to $H_{d}\left(\mathcal{D}_{a}\right)$. Moreover, the same reasoning holds too for the Dirichlet condition and one has $\mathbf{w}=\left(u_{0}, 0\right)$ on $\Gamma_{0}$.

It remains now to prove that $\mathbf{w}$ satisfies the variational formulation (2.19). Let us consider a test function $\mathbf{z}$ with compact support "below" $\Gamma_{a}$ and arbitrarily close to $\Gamma_{a}$. Thanks to the $L^{\infty}$ convergence of $a_{n}$ to $a$, one can find $n_{\varepsilon}$ such that the support of $\mathbf{z}$ lies below $\Gamma_{n}:=\Gamma_{a_{n}}$ for any $n \geq n_{\varepsilon}$ (see figure below). Let us write (2.19) for such a $n \geq n_{\varepsilon}$ :

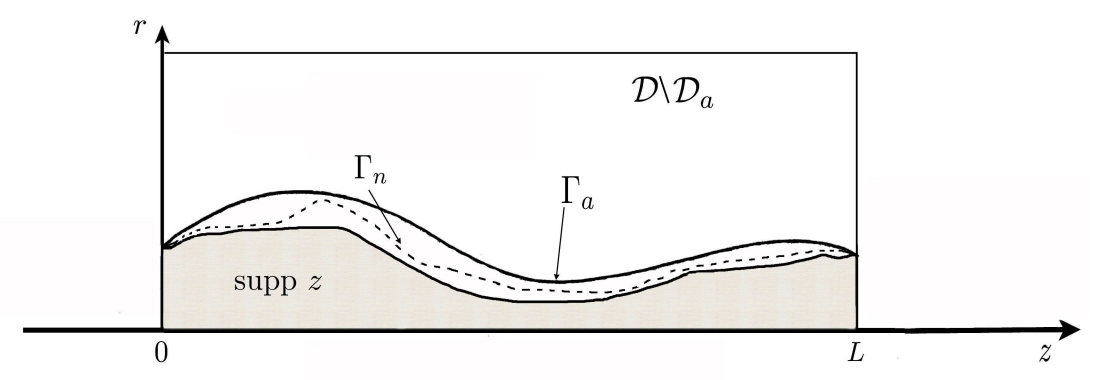

FIG. 3.1. Choice of test functions

$$
2 \mu \int_{\mathcal{D}}\left(\varepsilon_{2}\left(\mathbf{w}_{\mathbf{n}}\right): \varepsilon_{2}(\mathbf{z})+\frac{w_{2 n} z_{2}}{r^{2}}\right) r \mathrm{~d} r \mathrm{~d} z=0
$$

Using that the norms $H_{0}^{1}$ and $\|\cdot\|_{\varepsilon}$ are equivalent, and the fact that $\mathbf{w}_{\mathbf{n}}$ converges weakly- $H^{1}(\mathcal{D})$ and strongly- $L^{2}(\mathcal{D})$ to $\widetilde{\mathbf{w}}$, one deduces

$$
\int_{\mathcal{D}}\left(\varepsilon_{2}\left(\mathbf{w}_{\mathbf{n}}\right): \varepsilon_{2}(\mathbf{z})+\frac{w_{2 n} z_{2}}{r^{2}}\right) r \mathrm{~d} r \mathrm{~d} z \underset{n \rightarrow+\infty}{\longrightarrow} \int_{\mathcal{D}}\left(\varepsilon_{2}(\widetilde{\mathbf{w}}): \varepsilon_{2}(\mathbf{z})+\frac{\widetilde{w}_{2} z_{2}}{r^{2}}\right) r \mathrm{~d} r \mathrm{~d} z .
$$

In other words,

$$
\forall \mathbf{z} \in H_{d}\left(\mathcal{D}_{a}\right) \cap \mathcal{C}_{c}^{\infty}\left(\overline{\mathcal{D}_{a}} \backslash \Gamma_{a}\right), 2 \mu \int_{\mathcal{D}_{a}}\left(\varepsilon_{2}(\widetilde{\mathbf{w}}): \varepsilon_{2}(\mathbf{z})+\frac{\widetilde{w}_{2} z_{2}}{r^{2}}\right) r \mathrm{~d} r \mathrm{~d} z=0
$$

and $\widetilde{\mathbf{w}}=0$ on $\Gamma_{a}, \widetilde{\mathbf{w}}=\left(u_{0}, 0\right)$ on $\Gamma_{0}$. The conclusion follows, applying Theorem 2.6.

\section{Qualitative and quantitative properties of the optimum.}

4.1. Shape derivative for Problem (2.7) in the case where $\Omega$ is regular. We are now in position to define the derivative of the functional $J_{0}$ with respect to the shape $\Omega$. In this section, let us assume that $\Omega$ has a Lipschitz boundary, to ensure the differentiability of $J_{0}$ at $\Omega$. Let us consider a regular $\left(C^{1, \infty}\right.$ for instance) vector field $\mathbf{V}: \mathbb{R}^{3} \rightarrow \mathbb{R}^{3}$ with compact support inside the strip $\left\{0<x_{3}<L\right\}$. For $t$ small enough, we define $\Omega_{t}=(I+t \mathbf{V}) \Omega$, the image of $\Omega$ by a perturbation of identity and $f(t)=J_{0}\left(\Omega_{t}\right)$. We recall that the shape derivative of $J_{0}$ at $\Omega$ exists (already mentioned in $[10,17,18,15,26,29])$ and is $f^{\prime}(0)$. We will denote it by $\mathrm{d} J_{0}(\Omega ; \mathbf{V})$. To compute it, we first need to compute the derivative of the state equation, using the classical results on shape derivatives (see $[14,27,31]$ ). The material derivative of $(\mathbf{u}, p)$ denoted $(\dot{\mathbf{u}}, \dot{p})$ is 
the solution of the following linear system,

$$
\left\{\begin{aligned}
-\mu \Delta \dot{\mathbf{u}}+\nabla \dot{p}=0 & \text { in } \Omega_{a} \\
\nabla \cdot \dot{\mathbf{u}}=0 & \text { in } \Omega_{a} \\
\dot{\mathbf{u}}=-\frac{\partial \mathbf{u}}{\partial n}(\mathbf{V} \cdot \mathbf{n}) & \text { on } \Sigma_{a} \\
\dot{\mathbf{u}}=0 & \text { on } E_{a} \\
\sigma(\dot{\mathbf{u}}, \dot{p}) \cdot \mathbf{n}=0 & \text { on } S_{a}
\end{aligned}\right.
$$

The shape derivative with respect to the domain is classically given by the formula

$$
\mathrm{d} J_{0}(\Omega ; \mathbf{V})=4 \mu \int_{\Omega} \varepsilon(\dot{\mathbf{u}}): \varepsilon(\mathbf{u}) \mathrm{d} x+\int_{\partial \Omega}|\varepsilon(\mathbf{u})|^{2}(\mathbf{V} \cdot \mathbf{n}) \mathrm{d} s .
$$

In general, it is more convenient to work with another expression of the shape derivative and to write it as a distribution with support $\Sigma=\partial \Omega \backslash(E \cup S)$. For that purpose, we need in general to introduce an adjoint state. Nevertheless, the Stokes operator is self-adjoint and we will show that the adjoint problem of (2.4) is the same.

Proposition 4.1. Let $\Omega \in \mathcal{O}$, a domain with $C^{2}$ boundary. Then, the functional $J_{0}$ is shape-differentiable at $\Omega$ and one has

$$
\mathrm{d} J_{0}(\Omega ; \mathbf{V})=-2 \mu \int_{\partial \Omega}|\varepsilon(\mathbf{u})|^{2}(\mathbf{V} \cdot \mathbf{n}) \mathrm{d} s
$$

As a consequence, the first order optimality conditions for problem (2.7) write

$$
\Omega \text { solution of problem (2.7) with } C^{2} \text { boundary } \Rightarrow|\varepsilon(\mathbf{u})|^{2}=\text { constant on } \Sigma \text {. }
$$

Proof. The differentiability has been already studied, as mentioned above. Let us now prove (4.3). Since the boundary $\Sigma$ is assumed to be $C^{2}, \varepsilon(\mathbf{u})$ belongs to $L^{2}(\Sigma)$ and the normal vector $\mathbf{n}$ exists everywhere. Let us multiply equation (2.4) by $\dot{\mathbf{u}}$ and then integrate by part. We get

$$
\begin{aligned}
2 \mu \int_{\Omega} \varepsilon(\dot{\mathbf{u}}): \varepsilon(\mathbf{u}) \mathrm{d} x & =\int_{\partial \Omega} \sigma(\mathbf{u}, p) \mathbf{n} \cdot \dot{\mathbf{u}} \mathrm{d} s \\
& =\int_{\Sigma} p \mathbf{n} \cdot \frac{\partial \mathbf{u}}{\partial n}(\mathbf{V} \cdot \mathbf{n}) \mathrm{d} s-2 \mu \int_{\Sigma} \varepsilon(\mathbf{u}) \mathbf{n} \cdot \frac{\partial \mathbf{u}}{\partial n}(\mathbf{V} \cdot \mathbf{n}) \mathrm{d} s
\end{aligned}
$$

Since $\mathbf{u}$ is "divergence-free" and vanishes on $\Sigma$, we have on this boundary

- $\frac{\partial \mathbf{u}}{\partial n} \cdot \mathbf{n}=0$

- $\varepsilon(\mathbf{u}) \mathbf{n} \cdot \frac{\partial \mathbf{u}}{\partial n}=|\varepsilon(\mathbf{u})|^{2}$.

These remarks permit immediately to recover the expression of the shape derivative of $J_{0}$ given in (4.3) using in particular (4.2). Now let us write the first order optimality conditions. Because of the volume constraint, there exists a Lagrange multiplier $\lambda \in \mathbb{R}_{+}$such that

$$
-2 \mu \int_{\partial \Omega}|\varepsilon(\mathbf{u})|^{2}(\mathbf{V} \cdot \mathbf{n}) \mathrm{d} s=-2 \mu \lambda \int_{\Sigma}(\mathbf{V} \cdot \mathbf{n}) \mathrm{d} s .
$$

Since this equality holds for a generic element $\mathbf{V} \in C^{1, \infty}\left(\mathbb{R}^{3}, \mathbb{R}^{3}\right)$ with compact support inside the strip $\left\{0<x_{3}<L\right\}$, the first order optimality conditions (4.4) follow. 
In the case where the two disks $E$ and $S$ have the same radius $R>0$, the prescribed volume is equal to the one of the finite cylinder between the two disks $E$ and $S, \mathbf{h}$ is chosen so that the standard Poiseuille flow ${ }^{1}$ solves $(2.4)$ and if we assume that the flow at the inlet $E$ is parabolic, one could naturally think that the cylinder solves the shape optimization problem (2.7). Indeed, in this case, one has for $c<0$

$$
u_{0}=c\left(x_{1}^{2}+x_{2}^{2}-R^{2}\right), \varepsilon(\mathbf{u})=\left(\begin{array}{ccc}
0 & 0 & c x_{1} \\
0 & 0 & c x_{2} \\
c x_{1} & c x_{2} & 0
\end{array}\right),|\varepsilon(\mathbf{u})|^{2}=2 c^{2} R^{2} \text { on } \Gamma .
$$

The first order optimality conditions (4.4) are then satisfied. Nevertheless, the cylinder is not optimal for this particular choice of $\mathbf{h}$ as emphasizes [16, Theorem 2.5]. Furthermore, let us notice that the optimal domain $\Omega$ is solution of an overdetermined system, that is

$$
\left\{\begin{aligned}
-\mu \Delta \mathbf{u}+\nabla p=0 & \text { in } \Omega \\
\nabla \cdot \mathbf{u}=0 & \text { in } \Omega \\
\mathbf{u}=0 & \text { on } \Sigma \\
|\varepsilon(\mathbf{u})|^{2}=\mathrm{constant} & \text { on } \Sigma \\
\mathbf{u}=\mathbf{u}_{\mathbf{0}} & \text { on } E \\
\sigma(\mathbf{u}, p) \cdot \mathbf{n}=\mathbf{h} & \text { on } S .
\end{aligned}\right.
$$

The question of the determination of $\Omega$ (or some qualitative properties of $\Omega$ ) is very linked to the question of the unique continuation property for the Stokes system. Indeed, the optimal domain $\Omega$ satisfies a Cauchy system near the "free boundary" $\Sigma$. It may be noticed that, in dimension two, a positive answer was given in [28], but for generic domains only.

4.2. Shape derivative for the axisymmetric problem $(2.21)$ in the case $p \in[2,+\infty]$ and $a \in C^{2}([0, L])$. Let us define the derivative of the functional $J$ with respect to the shape represented by $a$. It is well known $[1,14]$ that assuming $a \in \mathcal{A}_{p}$ ensures the differentiability of $J$ at $\mathbf{w}_{\mathbf{a}}$ and the fact that $a$ is assumed furthermore to be in $C^{2}([0, L])$ justifies that $\mathbf{w}$ belongs to $\left[W^{2,2}\left(\mathcal{D}_{a}\right)\right]^{2}$ and permits to use integration by parts formula. Let us consider $\delta a \in W_{c}^{1, p}(0, L)$, a perturbation of $a$ with compact support, support which does not meet neither $\{z=0\}$ nor $\{z=L\}$. For $t$ small enough, we define $a_{t}=a+t \delta a$, the image of $a$ by a perturbation of the identity and $f(t)=J\left(\mathbf{w}_{\mathbf{a}_{\mathbf{t}}}\right)$. We recall that the shape derivative of $J$ at $\mathbf{w}_{\mathbf{a}}$ exists and is $f^{\prime}(0)$. In what follows, we will prefer the notation $J(a)$ to designate the functional $J\left(\mathbf{w}_{\mathbf{a}}\right)$; similarly $f^{\prime}(0)$ will be denoted (with a slight abuse of notations) $\mathrm{d} J(a ; \delta a)$. Let us first define the derivative of the state equation, using the classical calculus of variation results. The material derivative of $\mathbf{w}_{a}$ denoted $\dot{\mathbf{w}}$ is the solution of the linear system, written under variational formulation

$$
\forall \mathbf{z} \in H_{d}\left(\mathcal{D}_{a}\right) \cap \mathcal{C}_{c}^{\infty}\left(\overline{\mathcal{D}_{a}} \backslash \Gamma_{a}\right), 2 \mu \int_{\mathcal{D}_{a}}\left(\varepsilon_{2}(\dot{\mathbf{w}}): \varepsilon_{2}(\mathbf{z})+\frac{\dot{w}_{2} z_{2}}{r^{2}}\right) r \mathrm{~d} r \mathrm{~d} z=0,
$$

and $\dot{\mathbf{w}}=-\frac{\delta a(z)}{\sqrt{1+a^{\prime 2}(z)}} \frac{\partial \mathbf{w}_{a}}{\partial n}$ on $\Gamma_{a}, \dot{\mathbf{w}}=(0,0)$ on $\Gamma_{0}$. Indeed, coming back to the three dimensional representation of our shape optimization problem, following the notations summed up at the left hand side of Figure 2.1, that is easy to see that on $\Sigma_{a}$,

$$
\mathbf{n}=\frac{1}{\sqrt{1+a^{\prime 2}(z)}}\left(\begin{array}{c}
\cos \theta \\
\sin \theta \\
-a^{\prime}(z)
\end{array}\right)
$$

\footnotetext{
${ }^{1}$ The Poiseuille flow is such that $\mathbf{u}=\left(0,0, u_{0}(r)\right)$ for $(z, r) \in(0, L) \times\left(0, R_{0}\right)$ and $p$ is an affine function of the variable $z$.
} 
and the perturbation field applied at any point of the boundary $\Sigma_{a}$ is $\mathbf{V}=\left(\begin{array}{c}\delta a \cos \theta \\ \delta a \sin \theta \\ 0\end{array}\right)$. It is very common in shape optimization to differentiate a Dirichlet boundary condition with respect to the domain (see e.g. [4, Chapter 4] or [14, Chapter 5]), and one has

$$
\dot{\mathbf{w}}=-\frac{\partial \mathbf{w}_{a}}{\partial n}(\mathbf{V} \cdot \mathbf{n}) \text { on } \Gamma_{a},
$$

providing the expected result. Using relation (3.10), we claim that the shape derivative with respect to the domain is given by the formula

$$
\begin{aligned}
\mathrm{d} J(a ; \delta a) & =8 \pi \mu \int_{\mathcal{D}_{a}}\left[\varepsilon_{2}(\dot{\mathbf{w}}): \varepsilon_{2}\left(\mathbf{w}_{a}\right)+\frac{\dot{w}_{2} w_{a, 2}}{r^{2}}\right] r \mathrm{~d} r \mathrm{~d} z+4 \pi \mu \int_{\Gamma_{a}}\left|\varepsilon_{2}\left(\mathbf{w}_{\mathbf{a}}\right)\right|^{2}\left(\mathbf{V}_{\mathbf{2}} \cdot \mathbf{n}_{\mathbf{2}}\right) \mathrm{d} \sigma \\
& =8 \pi \mu \int_{\partial \mathcal{D}_{a}} \varepsilon_{2}\left(\mathbf{w}_{a}\right) \mathbf{n}_{\mathbf{2}} \cdot \dot{\mathbf{w}} \mathrm{d} \sigma+4 \pi \mu \int_{\Gamma_{a}}\left|\varepsilon_{2}\left(\mathbf{w}_{\mathbf{a}}\right)\right|^{2}\left(\mathbf{V}_{\mathbf{2}} \cdot \mathbf{n}_{\mathbf{2}}\right) \mathrm{d} \sigma
\end{aligned}
$$

where $\mathbf{n}_{\mathbf{2}}=\frac{1}{\sqrt{1+a^{\prime 2}}}\left(\begin{array}{c}-a^{\prime}(z) \\ 1\end{array}\right), \mathbf{V}_{\mathbf{2}}=\left(\begin{array}{c}0 \\ \delta a(z)\end{array}\right)$, and $d \sigma$ stands for the curvilinear measure on $\Gamma_{a}$, that is $\mathrm{d} \sigma=\sqrt{1+a^{\prime 2}(z)} \mathrm{d} z$.

Now, since $\dot{\mathbf{w}}=-\frac{\partial \mathbf{w}_{\mathbf{a}}}{\partial n}\left(\mathbf{V}_{\mathbf{2}} \cdot \mathbf{n}_{\mathbf{2}}\right)$ and $\varepsilon_{2}\left(\mathbf{w}_{a}\right) \mathbf{n}_{\mathbf{2}} \cdot \frac{\partial \mathbf{w}_{\mathbf{a}}}{\partial n}=\left|\varepsilon_{2}\left(\mathbf{w}_{a}\right)\right|^{2}$ (due to the fact that $\mathbf{w}_{\mathbf{a}}$ vanishes on $\Gamma_{a}$ ), one gets

$$
\mathrm{d} J(a ; \delta a)=-4 \pi \mu \int_{\Gamma_{a}}\left|\varepsilon_{2}\left(\mathbf{w}_{\mathbf{a}}\right)\right|^{2}\left(\mathbf{V}_{\mathbf{2}} \cdot \mathbf{n}_{\mathbf{2}}\right) \mathrm{d} \sigma .
$$

To rewrite this shape derivative as an integral with respect to the variable $z$, we use the previous expressions of $\mathbf{V}_{\mathbf{2}}$ and $\mathbf{n}_{\mathbf{2}}$. This is done in the following proposition.

Proposition 4.2. Let $a \in C^{2}([0, L])$. Then, the functional $J$ is shape-differentiable at $\mathbf{w}_{\mathbf{a}}$ and one has

$$
\mathrm{d} J(a ; \delta a)=-4 \pi \mu \int_{0}^{L}\left|\varepsilon_{2}\left(\mathbf{w}_{\mathbf{a}}\right)\right|^{2} \delta a(z) \mathrm{d} z .
$$

As a consequence, if $a^{*} \in C^{2}([0, L])$ is the optimal solution to problem (2.21), then for any $a \in \mathcal{U}_{p}$,

$$
\int_{0}^{L}\left|\varepsilon_{2}\left(\mathbf{w}_{\mathbf{a}^{*}}\right)\right|^{2}\left(a(z)-a^{*}(z)\right) \mathrm{d} z \leq 0
$$

4.3. Explicit solution of problem (2.21) without regularity assumption on $a$. This section is devoted to the proof of a useful property of the functional $J$, that will appear essential to explore the necessary first order optimality conditions for problem (2.21). In particular, we will give the explicit solution of this optimization problem in the case $h_{0}=h_{L}=0$ where no condition is imposed on the inlet and outlet.

For that purpose, let us use the natural order relation $\leq$ defined for two elements of the set $\mathcal{A}_{p}, p \in[2,+\infty]$, by

$$
a \leq b \Longleftrightarrow a(z) \leq b(z) \text {, for } z \in(0, L)
$$


Proposition 4.3. The functional $a \in \mathcal{A}_{p} \mapsto J(a)$ is a strictly decreasing function with respect to the order relation $\leq$.

Proof. The fact that $J$ is monotone decreasing is easy to see. It suffices to adapt the end of the proof of Theorem 2.3, noticing that

$$
J(a)=2 \mu \min _{\mathbf{w} \in H_{2, \mathrm{div}}\left(\mathcal{D}_{a}\right)} \int_{\mathcal{D}_{a}}\left[\left|\varepsilon_{2}(\mathbf{w})\right|^{2}+\frac{w_{2}^{2}}{r^{2}}\right] r \mathrm{~d} r \mathrm{~d} z,
$$

where $H_{2, \operatorname{div}}\left(\mathcal{D}_{a}\right)=\left\{\mathbf{w} \in\left[W^{1,2}\left(\mathcal{D}_{a}\right)\right]^{2} \mid \mathbf{w}\right.$ satisfies $(3.7), \mathbf{w}_{\left.\right|_{\Gamma_{0}}}=\left(u_{0}, 0\right)$ and $\left.\mathbf{w}_{\left.\right|_{\Gamma_{a}}}=0\right\}$. The monotonicity of $J$ follows then from the inclusion of the Sobolev spaces: $a \leq b \Longrightarrow H_{2, \operatorname{div}}\left(\mathcal{D}_{a}\right) \subset$ $H_{2, \operatorname{div}}\left(\mathcal{D}_{b}\right)$.

It remains to prove the strict character of the decreasing property for $J$. Let us argue by contradiction, considering $a \in \mathcal{A}_{p}$ and assuming that there exists $b \in \mathcal{A}_{p}$ such that $a \leq b$ and $J(b)=J(a)$. Let us denote by $\mathbf{w}_{\mathbf{a}}$ the unique (see Lemma 2.2) minimizer that realizes $J(a)$ and by $\mathbf{w}_{\mathbf{b}}$ the unique minimizer that realizes $J(b)$. We will again designate by $\mathbf{w}_{\mathbf{a}}$ the extension by 0 of this function to the whole domain $\mathcal{D}_{b}$. In particular, it is easy to see that this extension by continuity belongs to $\left[W^{1,2}\left(\mathcal{D}_{b}\right)\right]^{2}$ and satisfies again $(3.7)$. Hence, because of the uniqueness of the minimizer (underlined in Lemma 2.2), necessarily $\mathbf{w}_{\mathbf{a}}=\mathbf{w}_{\mathbf{b}}$. It implies that at the same time, the minimizer $\mathbf{w}_{\mathbf{b}}$ is solution of a Stokes system and vanishes in an open ball included in $\mathcal{D}_{b} \backslash \mathcal{D}_{a}$. By virtue of the analyticity of the Stokes operator (see for instance [20]), this is absurd, and one deduces that the inequality between $J(a)$ and $J(b)$ is strict.

A direct consequence of Proposition 4.3 is the fact that our shape optimization problem with Stokes constraints rewrites as a purely geometrical problem. As a result, we immediately deduce from this result the following corollary.

COROLlary 4.4. In the case $h_{0}=h_{L}=0$, the optimal solution of problem (2.21) is

$$
a^{*}=a_{1}
$$

Proof. Proposition 4.3 yields that the unique solution to

$$
\left\{\begin{array}{l}
\max J(a) \\
a \in \mathcal{A}_{p}
\end{array}\right.
$$

is $a^{*}=a_{1}$. Since $a^{*}$ is feasible for problem (2.21), the conclusion follows. follows

Let us now investigate the case where $p \in[2,+\infty]$ and the inlet and outlet are prescribed as

$$
h_{0}(x)=x-\overline{a_{0}} \text { and } h_{L}(x)=x-\overline{a_{L}},
$$

with $\left(\overline{a_{0}}, \overline{a_{1}}\right) \in\left(a_{0}, a_{1}\right)^{2}$. So far, the cylinder is not the optimal shape any longer [16]. It may be noticed that taking into account some inequality constraints of the kind $h_{0}(a(0)) \leq 0$ and $h_{L}(a(L)) \leq 0$ would be very easy since, because of Proposition 4.3 , these constraints would be reached, so that we would be led to study the case we investigate now. Notice that, since $a$ is a non negative function, finding the maximal element in a given class for the order relation $\leq$ is equivalent for instance to the maximization of the $L^{1}$ norm of $a$. Therefore, $a^{*}$ solving problem 
(2.21) is in fact solution of

$$
\left\{\begin{array}{l}
\max \int_{0}^{L} a(z) \mathrm{d} z \\
a \in W^{1, p}(0, L) \\
\left\|a^{\prime}\right\|_{p} \leq M \text { and } a_{0} \leq a(z) \leq a_{1} \text { for } z \in(0, L) \\
a(0)=\overline{a_{0}} \text { and } a(L)=\overline{a_{L}} .
\end{array}\right.
$$

Because of the simplicity of this new geometrical problem, we are in position to give a quasiexplicit expression of its solution $a^{*}$ in the case $p<+\infty$ and the explicit expression of $a^{*}$ in the case $p=+\infty$.

THEOREM 4.5. Let $h_{0}$ and $h_{L}$ given by (4.8).

1. Case $p<+\infty$. Problem (2.21) has a unique solution $a^{*}$ that is the solution of problem (4.9). Moreover,

- There exists $\left(z_{1}, z_{2}\right) \in[0, L]^{2}, z_{1} \leq z_{2}$ such that $a^{*}$ is strictly monotone increasing and concave on $\left(0, z_{1}\right)$, constant equal to $a_{1}$ on $\left(z_{1}, z_{2}\right)$ and strictly monotone decreasing and convex on $\left(z_{2}, L\right)$.

- One has $\left\|a^{* \prime}\right\|_{p}=M$.

- There exists $\left(m, c_{1}, c_{2}\right) \in \mathbb{R}_{+}^{3}$ such that

$$
a^{*}(z)= \begin{cases}\overline{a_{0}}+m\left(\left(z+c_{1}\right)^{\frac{p}{p-1}}-c_{1}^{\frac{p}{p-1}}\right) & \text { if } z \in\left(0, z_{1}\right) \\ a_{1} & \text { if } z \in\left(z_{1}, z_{2}\right) \\ \overline{a_{L}}+m\left(c_{2}^{\frac{p}{p-1}}-\left(z-L+c_{2}\right)^{\frac{p}{p-1}}\right) & \text { if } z \in\left(z_{2}, L\right) .\end{cases}
$$

2. Case $p=+\infty$. Problem (2.21) has a unique solution $a^{*}$ that is the solution of problem (4.9). More precisely:

(a) Case $\min \left(\overline{a_{0}}, \overline{a_{1}}\right)>a_{1}-L M$. The solution $a^{*}$ is defined by

$$
a^{*}(z)= \begin{cases}M z+\overline{a_{0}} & \text { if } z \in\left(0, \frac{a_{1}-\overline{a_{0}}}{-\bar{M}}\right) \\ a_{1} & \text { if } z \in\left(\frac{a_{1}}{M}, L-\frac{a_{1}-\overline{a_{L}}}{M}\right) \\ -M(z-L)+\overline{a_{L}} & \text { if } z \in\left(L-\frac{a_{1}-\overline{a_{L}}}{M}, L\right) .\end{cases}
$$

(b) Case $\min \left(\overline{a_{0}}, \overline{a_{1}}\right) \leq a_{1}-L M$. The solution $a^{*}$ is defined by

$$
a^{*}(z)= \begin{cases}M z+\overline{a_{0}} & \text { if } z \in\left(0, \frac{\overline{a_{1}}-\overline{a_{0}}+M L}{2 M}\right) \\ -M(z-L)+\overline{a_{L}} & \text { if } z \in\left(\frac{\overline{\bar{a}}-\overline{a_{0}}+M L}{2 M}, L\right) .\end{cases}
$$

Proof. In this proof, we denote by $F(a)$ the quantity $F(a)=\int_{0}^{L} a(z) \mathrm{d} z$.

1. Case $p<+\infty$. This proof will be detailed into several steps. Let us recall that the admissible set is convex and is denoted $\mathcal{U}_{p}$.

- Step 1: existence of a solution $a^{*}$.

Let $\left(a_{n}\right)_{n \in \mathbb{N}}$ be a maximizing sequence for problem (4.9). $\left(a_{n}\right)_{n \in \mathbb{N}}$ is uniformly bounded in $W^{1, p}(0, L)$ because of the $L^{\infty}$-constraint on $a$ and the $L^{p}$-constraint on $a^{\prime}$. Therefore, there exists $a^{*}$ such that, up to a subsequence,

$$
a_{n} \stackrel{L^{\infty} \stackrel{(0, L)}{\rightarrow}}{\rightarrow} a^{*} \text { and } a_{n} \stackrel{W^{1, p}(0, L)}{\rightarrow} a^{*} \text { as } n \rightarrow+\infty .
$$


Furthermore, the weak- $W^{1, p}$ convergence of $\left(a_{n}\right)_{n \in \mathbb{N}}$ to $a^{*}$ implies

$$
\left\|a^{* \prime}\right\|_{p} \leq \liminf _{n \rightarrow+\infty}\left\|a_{n}^{\prime}\right\|_{p} \leq M
$$

This yields that $\left(J\left(a_{n}\right)\right)_{n \in \mathbb{N}}$ converges to $J\left(a^{*}\right)$ as $n \rightarrow+\infty$ and that $a^{*}$ belongs to $\mathcal{U}_{p}$. The existence follows.

- Step 2: the constraint " $\left\|a^{* \prime}\right\|_{p} \leq M$ " is reached.

Let $\eta>0$, small enough. Since $a^{*}$ is continuous, and since $a^{*}(0)=\overline{a_{0}}<a_{1}$, there exists an open subset $\omega$ on which $\overline{a_{0}}+\eta \leq a^{*}(z) \leq a_{1}-\eta$ for $z \in \omega$. Let us argue by contradiction, assuming that the constraint " $\left\|a^{* \prime}\right\|_{p} \leq M$ " is not active. For any admissible perturbation $\delta a$ with compact support included in $\omega$ (the existence of such perturbations $\delta a$ is obvious), one can write the first order optimality condition. One has for $\kappa>0$ small enough, $F\left(a^{*}+\kappa \delta a\right) \geq F\left(a^{*}\right)$, where $F\left(a^{*}\right)=\int_{0}^{L} a^{*}(z) \mathrm{d} z$. Dividing the previous inequality by $\kappa$ and making $\kappa$ go to zero yields

$$
\mathrm{d} F\left(a^{*} ; \delta a\right) \geq 0 \text {. }
$$

Furthermore, if $\kappa$ is small enough, perturbations $a \pm \kappa \delta a$ are admissible and the same reasoning as before proves that the derivative of $F$ at $a^{*}$ in direction $\delta a$ vanishes so that

$$
\mathrm{d} F(a ; \delta a)=\int_{\omega} \delta a(z) \mathrm{d} z=0 .
$$

Any choice of admissible $\delta a$ with nonzero mean provides a contradiction.

- Step 3: the optimum $a^{*}$ is unique.

The reason of this uniqueness comes from the fact that the convex functional $F$ defined on $\mathcal{U}_{p}$ is strictly locally convex around the optimum $a^{*}$. Indeed, let us assume the existence of two elements $a_{1}$ and $a_{2}$ of $\mathcal{U}_{p}$ such that

$$
\max _{a \in \mathcal{U}_{p}} F(a)=F\left(a_{1}\right)=F\left(a_{2}\right) .
$$

Because of the linearity of $F$, for any $t \in(0,1)$ the function $a_{t}=t a_{1}+(1-t) a_{2}$ is also a solution of problem (4.9). As a consequence of Step 2, we necessarily have $\left\|a_{t}^{\prime}\right\|_{p}^{p}=M^{p}$, i.e. $\left\|t a_{1}^{\prime}+(1-t) a_{2}^{\prime}\right\|_{p}^{p}=M^{p}$. Moreover, $t\left\|a_{1}^{\prime}\right\|_{p}^{p}+(1-t)\left\|a_{2}^{\prime}\right\|_{p}^{p}=M^{p}$, and since $p \geq 2$, the function $x \mapsto x^{p}$ is strictly convex on $\mathbb{R}_{+}$. Hence, by virtue of Jensen's inequality, $a_{1}=a_{2}$ providing the expected result.

- Step 4: profile of the optimum $a^{*}$.

Let us notice that the number of connected components of the set $\left\{a=a_{1}\right\}$ is, at most equal to 1 . Indeed, assume that the set $\left\{a^{*}=a_{1}\right\}$ has two disjoint connected components $\omega_{1}$ and $\omega_{2}$, and that there exists a set of nonzero measure between these two sets on which $a \leq a_{1}-\eta$ for a given $\eta>0$ small enough. Let us denote by $\bar{\omega}$ the convex hull of $\omega_{1}$ and $\omega_{2}$. That is easy to see that we strictly improve the objective function $F$ replacing $a^{*}$ by $\bar{a}$ such that

$$
\bar{a}(z)= \begin{cases}a_{1} & \text { if } z \in \bar{\omega} \\ a^{*}(z) & \text { if } z \notin \bar{\omega},\end{cases}
$$

in other words, $F(\bar{a})>F\left(a^{*}\right)$, which contradicts the optimality of $a^{*}$. As a consequence, there exists $\left(z_{1}, z_{2}\right) \in[0, L]^{2}, z_{1} \leq z_{2}$ such that $a^{*}$ constant equal to $a_{1}$ on 
$\left(z_{1}, z_{2}\right)$ and $a^{*}<a_{1}$ on $[0, L] \backslash\left[z_{1}, z_{2}\right]$.

Let us now prove that $a^{*}$ is monotone increasing on $\left(0, z_{1}\right)$. We denote by $a_{\left(0, z_{1}\right)}^{*}$, the restriction of $a^{*}$ on $\left(0, z_{1}\right)$. For that purpose, let us introduce $a^{i}$, the monotonically increasing rearrangement of $a_{\left(0, z_{1}\right)}^{*}$, that is defined by

$$
\forall z \in\left(0, z_{1}\right), a^{i}\left(z_{1}-z\right)=\inf \{t \in \mathbb{R}, m(t) \leq z\},
$$

where $m$ denotes the distribution function of $a_{\left(0, z_{1}\right)}^{*}$, i.e. $m: t \in \mathbb{R} \mapsto$ meas $\left(\left\{a_{\left(0, z_{1}\right)}^{*}>\right.\right.$ $t\})$. Notice that, since $a_{\left(0, z_{1}\right)}^{*}$ belongs to $W^{1, p}$ on the connected set $\left(0, z_{1}\right)$, then this is also the case for $a^{i}$ (see for instance $[19,14]$ ). By virtue of Polyà's inequality, $\left\|a^{i^{\prime}}\right\|_{p} \leq\left\|a_{\left(0, z_{1}\right)}^{*}{ }^{\prime}\right\|_{p} \leq M$ and because of the equimeasurability property of the monotone rearrangement, $\int_{0}^{z_{1}} a^{i}(z) \mathrm{d} z=\int_{0}^{z_{1}} a^{*}(z) \mathrm{d} z$. Denoting again by $a^{i}$ the extension of the function $a^{i}$ to $(0, L)$ satisfying $a^{i}=a^{*}$ on $\left(z_{1}, L\right)$, it follows from the previous remarks that $a^{i}$ and $a^{i}$ both realize the maximum of $F$ over the set $\mathcal{U}_{p}$. This is in contradiction with the uniqueness result of Step 3, whence the conclusion. An adaptation of the previous reasoning proves that $a^{*}$ is monotone decreasing on $\left(z_{2}, L\right)$.

- Step 5: first order optimality conditions.

Because of the previous conclusions, one can choose $\eta>0$ and $\omega \subset\left(0, z_{1}\right)$, open, such that for any $z \in \omega, \overline{a_{0}}+\eta \leq a^{*}(z) \leq a_{1}-\eta$. Since the global $L^{p}$-constraint is attained at the optimum, the first order optimality conditions yield in particular the existence of a Lagrange multiplier $\lambda \geq 0^{2}$ such that

$$
\forall \delta a \in W_{c}^{1, p}(\omega), \int_{\omega} \delta a(z) \mathrm{d} z=-\lambda p \int_{\omega}\left(a^{* \prime}\right)^{p-1}(z)(\delta a)^{\prime}(z) \mathrm{d} z,
$$

where $W_{c}^{1, p}(\omega)$ denotes the set of functions living in the Sobolev space $W^{1, p}(0, L)$, with compact support included in $\omega$. Now, let $x_{1}$ and $x_{2}$ be two elements of $\omega, \varepsilon>0$ small enough and let us consider particular perturbations $\delta a$ with support $\left(x_{1}, x_{2}\right)$ such that

$$
\delta a(z)= \begin{cases}x-x_{1} & \text { if } z \in\left(x_{1}, x_{1}+\varepsilon\right) \\ \varepsilon & \text { if } z \in\left(x_{1}+\varepsilon, x_{2}-\varepsilon\right) \\ x_{2}-x & \text { if } z \in\left(x_{2}-\varepsilon, x_{2}\right)\end{cases}
$$

Hence, we clearly have $(\delta a)^{\prime}(z)=\chi_{\left[x_{1}, x_{1}+\varepsilon\right]}-\chi_{\left[x_{2}, x_{2}+\varepsilon\right]}$. The optimality condition rewrites then

$$
\varepsilon\left(x_{2}-x_{1}-\varepsilon\right)=\lambda p\left(\int_{\left[x_{1}, x_{1}+\varepsilon\right]}\left(a^{* \prime}\right)^{p-1}(z) \mathrm{d} z-\int_{\left[x_{2}-\varepsilon, x_{2}\right]}\left(a^{* \prime}\right)^{p-1}(z) \mathrm{d} z\right) .
$$

Let us divide this identity by $\varepsilon$ and then, make $\varepsilon$ tend to 0 . The Lebesgue's density theorem yields

$$
\left(a^{* \prime}\right)^{p-1}\left(x_{2}\right)-\left(a^{* \prime}\right)^{p-1}\left(x_{1}\right)=\lambda p\left(x_{2}-x_{1}\right) .
$$

Since $x_{1}$ and $x_{2}$ are chosen arbitrarily, this identity implies obviously that $\lambda \neq 0$ (else, $a^{*}$ would be constant on $\left(0, z_{1}\right)$ which is not compatible with $a(0)=a_{0}$ and

\footnotetext{
${ }^{2} \lambda$ is associated with the global inequality constraint on $a^{\prime}$ (that is in fact an equality constraint), whence its sign
} 
$\left.a\left(z_{1}\right)=a_{1}\right)$, that $a^{* \prime}$ cannot vanish on $\left(0, z_{1}\right)$, that $a^{* \prime}$ is a monotone decreasing function, so that $a^{*}$ is concave on $\left(0, z_{1}\right)$, that $a^{* \prime}$ is differentiable on $\left(0, z_{1}\right)$ and that

$$
(p-1) a^{* \prime \prime}(z)\left(a^{* \prime}(z)\right)^{p-2}=\frac{1}{\lambda p}, z \in\left(0, z_{1}\right) .
$$

Notice that one can establish in the same way that

$$
-(p-1) a^{* \prime \prime}(z)\left(-a^{* \prime}(z)\right)^{p-2}=\frac{1}{\lambda p}, z \in\left(z_{2}, L\right) .
$$

Now, the expressions given in the statement of the theorem follows directly from the integration of these two differential equations. These computations are a bit tedious but easy and are left to the reader.

2. Case $p=+\infty$. The existence of a solution $a^{*}$ follows from a direct adaptation of the previous case. The case " $p=+\infty$ " can been studied by a geometrical way. Indeed, let us assume that there exists a nonzero measure subset of $(0, L)$ on which the constraints $\left|a^{\prime}\right| \leq M$ and $a \leq a_{1}$ are not active. Hence, it is easy to see that one can find a perturbation $\delta a$ with compact support in $\omega$ such that $\left|(a+\delta a)^{\prime}\right| \leq M$ on $\omega$ and $\int_{0}^{L}(a(z)+\delta a(z)) \mathrm{d} z>$ $\int_{0}^{L} a(z) \mathrm{d} z$. It proves that $(0, L)=\left\{a=a_{1}\right\} \cup\left\{\left|a^{\prime}\right|=M\right\}$. The same argument as in the previous case proves that the number of connected components of the set $\left\{a=a_{1}\right\}$ is at most 1. Therefore, there exists $\left(z_{1}, z_{2}\right) \in[0, L]^{2}, z_{1} \leq z_{2}$ such that $a^{*}$ is affine increasing on $\left(0, z_{1}\right)$, constant equal to $a_{1}$ on $\left(z_{1}, z_{2}\right)$ and affine decreasing on $\left(z_{2}, L\right)$. The end of the proof is a direct calculus.

REMARK 3. Unfortunately, we are not in position to give an explicit expression of the constants that appear in the expression of $a^{*}$ given in the first case of Theorem 4.5, since these constants are solutions of strongly nonlinear equations resulting from the fact that $a^{*}$ belongs to $\mathcal{U}_{p}$ and from the quasi-explicit expression of $a^{*}$ given in this theorem.

REMARK 4. Role of the global or pointwise constraint on $a^{\prime}$. If one neglects the constraint on $a^{\prime}$ in problem (4.9), we claim that there does not exist a solution anymore. Nevertheless, there exists a maximizing sequence $\left(a_{n}\right)_{n \in \mathbb{N}}$ such that $\left(J\left(a_{n}\right)\right)_{n \in \mathbb{N}}$ converges to La $a_{1}$, although $a_{n}(0)=\overline{a_{0}}$ and $a_{n}(L)=\overline{a_{L}}$. Indeed, let us denote by $c:[0,1] \rightarrow[0,1]$, the Cantor function, also referred to as the Devil's staircase. Let us recall that $c$ is continuous, but not absolutely continuous and has zero derivative almost everywhere. Now, considering as minimizing sequence

$$
\forall n \in \mathbb{N}, a_{n}(z)= \begin{cases}\overline{a_{0}}+\left(a_{1}-\overline{a_{0}}\right) c(n z) & \text { if } z \in(0,1 / n) \\ a_{1} & \text { if } z \in(1 / n, L-1 / n) \\ \overline{a_{1}}+\left(a_{1}-\overline{a_{1}}\right) c(n(L-z)) & \text { if } z \in(L-1 / n, L),\end{cases}
$$

that is easy to verify the convergence result mentioned above.

In [16] was investigated the question of the optimality of the cylinder, with a volume equality constraint on set of admissible domains and a negative answer was given. Intuitively and from a physical point of view, an explanation of this non optimality comes from the fact that the considered shape optimization problem was too constrained. Indeed, not only the volume of the admissible domains was prescribed, but also some particular "outlet pressure conditions", that strongly influenced the optimal shape. In problem (2.21), the situation is quite different, since only global $W^{1, p}$-conditions on $a$ and $L^{p}$-conditions on $a^{\prime}$ are imposed, that only provides an upper bound on the volume of the admissible domains. In the following section, we wonder, a bit as in [16], what become the optimal graph of the function $a^{*}$ with an additional area constraint forbidding the situations described above, $M$ being a positive arbitrarily large constant. 
4.4. Problem (2.21) with an additional area constraint. Let us introduce the functional $\mathrm{Vol}^{3}$ defined by for any $a \in \mathcal{A}_{p}$ by

$$
\operatorname{Vol}(a)=\int_{0}^{L} a(z) \mathrm{d} z .
$$

The quantity $\operatorname{Vol}(a)$ stands for the total measure of the two dimensional domain $\mathcal{D}_{a}$. Let $V_{0}>L a_{0}$. We consider the new optimization problem

$$
\left\{\begin{array}{l}
\min J(a) \\
a \in \mathcal{U}_{p} \\
\operatorname{Vol}(a)=V_{0} .
\end{array}\right.
$$

If we had considered, instead of problem (4.10), problem (2.21) with an inequality constraint on the volume, we would have obtained the same solutions since, by virtue of Proposition 4.3 , the constraint is necessarily active at the optimum.

Since the functional Vol is obviously continuous for the usual strong $L^{\infty}(0, L)$-topology, we derive from Theorem 2.7 the following corollary.

Corollary 4.6. Let $p \in[2,+\infty]$. Problem (4.10) has (at least) a solution $a^{*}$.

Finally, let us notice that the main result of [16] yields a non trivial information on the solution of problem (4.10) for a particular choice of boundary condition at the outlet that does unfortunately not apply in our case. Indeed, a condition of the kind $\sigma_{2}(\mathbf{w}, \widetilde{p}) \mathbf{n}=\mathbf{h}$ with $\mathbf{h}=\left(-p_{1}, 2 \mu r\right)$ is considered,in [16] so that the standard Poiseuille flow solves System (2.9) whereas in our case, $\mathbf{h}=(0,0)$ and the analytic expressions of the flow $\mathbf{w}$ and the pressure $\widetilde{p}$ are not known. We are all the same in position to prove a similar theorem in our case.

TheOREM 4.7. Let $p \in[2,+\infty]$. Assume that $a(0)=a(L), V_{0}=L a^{2}(0)$ and $u_{0}^{\prime}(a(0)) \neq 0$ (this is in particular the case if $u_{0}(r)=c\left(R^{2}-r^{2}\right)$ with $c>0$ for instance). Then, the constant function $a=a(0)$ does not solve problem (4.10).

Proof. Let us set $R_{0}=a(0)=a(L)$ and $a$ the constant solution equal everywhere to $R_{0}$. We will argue by contradiction, assuming that the constant function $a=R_{0}$ solves the shape optimization problem (4.10). First notice that the first order optimality condition writes $\left|\varepsilon_{2}(\mathbf{w})\right|^{2}=$ constant on $\Gamma_{a}$. Because of the divergence condition, $\left.\frac{\partial w_{2}}{\partial n}\right|_{\Gamma_{a}}=\frac{\partial w_{2}}{\partial r_{\Gamma_{a}}}=-\left.\frac{\partial w_{1}}{\partial z}\right|_{\Gamma_{a}}=0$. Therefore, the first order optimality condition leads to the existence of a constant $\xi \in \mathbb{R}$ such that

$$
\frac{\partial w_{1}}{\partial n}=\xi \text { on } \Gamma_{a}
$$

Let us consider the three dimensional Stokes system (2.4), before taking into account the symmetry of the solutions and rewriting this system into (2.9) (this point of view appears simpler for the following manipulation). Then, applying the divergence operator to the main equation proves that the pressure $p$ is a harmonic function. Integrating the Laplacian of $p$ on the domain $\Omega_{a}$ leads to the relation

$$
\int_{\Gamma_{a}} \frac{\partial p}{\partial n}=0
$$

\footnotetext{
${ }^{3}$ The so-called volume constraint is in fact an area constraint since we reduced the $3 \mathrm{D}$ problem to a $2 \mathrm{D}-\mathrm{one}$ thanks to the symmetry. However, we use the same terminology to keep in mind that the problem is generically $3 \mathrm{D}$.
} 
Using the symmetry of the function $p$ underlined in Proposition 2.4, this implies

$$
\int_{\Gamma_{0}} \frac{\partial \widetilde{p}}{\partial z}=\int_{\Gamma_{L}} \frac{\partial \widetilde{p}}{\partial z}
$$

Indeed, from the cylindrical symmetry underlined in Proposition 2.4 and from (4.11), $\int_{\Gamma_{0}} \frac{\partial \widetilde{p}}{\partial z}+$ $\int_{\Gamma_{a}} \frac{\partial \widetilde{p}}{\partial r}=\int_{\Gamma_{L}} \frac{\partial \widetilde{p}}{\partial z}$. Let $r$ go to $R_{0}$ in the partial differential equation of (2.9) with solution $w_{1}$ yields

$$
\left.\frac{1}{\mu} \frac{\partial \widetilde{p}}{\partial r}\right|_{\Gamma_{a}}=\left.\frac{\partial^{2} w_{2}}{\partial r^{2}}\right|_{\Gamma_{a}},
$$

since $w_{2}$ vanishes on $\Gamma_{a}$ so that all the derivatives of $w_{2}$ with respect to $z$ vanish on $\Gamma_{a}$ and we have already seen that $\left.\frac{\partial w_{2}}{\partial r}\right|_{\Gamma_{a}}=0$. Now, differentiating the "divergence-free" condition with respect to $r$ and making $r \rightarrow R_{0}$ yields

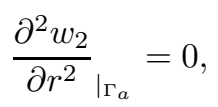

since the function $\frac{\partial w_{1}}{\partial r}$ remains constant on $\Gamma_{a}$ (optimality condition).

The end of the proof consists in computing each integral $\int_{\Gamma_{0}} \frac{\partial \widetilde{p}}{\partial z}, \int_{\Gamma_{L}} \frac{\partial \widetilde{p}}{\partial z}$, which will lead to a contradiction since we will obtain two different values.

- Computation of the integral $\int_{\Gamma_{0}} \frac{\partial \widetilde{p}}{\partial z}$.

Let us differentiate the "divergence-free" relation with respect to $z$. We get

$$
\frac{\partial^{2} w_{1}}{\partial z^{2}}+\frac{1}{r} \frac{\partial}{\partial r}\left(r \frac{\partial w_{2}}{\partial z}\right)=0 .
$$

Let us now consider the partial differential equation in $w_{1}$ and integrate it on $\Gamma_{0}$. Using the last above identity, we obtain

$$
\begin{aligned}
\frac{1}{\mu} \int_{\Gamma_{0}} \frac{\partial \widetilde{p}}{\partial z} & =\int_{\Gamma_{0}}\left[\frac{1}{r} \frac{\partial}{\partial r}\left(r \frac{\partial w_{1}}{\partial r}\right)+\frac{\partial^{2} w_{1}}{\partial z^{2}}\right] r \mathrm{~d} r \\
& =\left[r\left(\frac{\partial w_{1}}{\partial r}-\frac{\partial w_{2}}{\partial z}\right)\right]_{0}^{R_{0}} \\
& =R_{0} u^{\prime}\left(R_{0}\right)-R_{0} \frac{\partial w_{2}}{\partial z}\left(0, R_{0}\right) .
\end{aligned}
$$

Using the fact that the partial differential equation in $w_{1}$ is set on a very regular domain (a rectangle), one has

$$
\frac{\partial w_{2}}{\partial z}\left(0, R_{0}\right)={\frac{\partial w_{2}}{\partial z}}_{\Gamma_{0} \cap \Gamma_{a}}=0 .
$$

Finally,

$$
\frac{1}{\mu} \int_{\Gamma_{0}} \frac{\partial \widetilde{p}}{\partial z}=R_{0} u^{\prime}\left(R_{0}\right) .
$$

- Computation of the integral $\int_{\Gamma_{L}} \frac{\partial \widetilde{p}}{\partial z}$.

Let us differentiate the boundary condition on $\Gamma_{L}$ with respect to the variable $r$. We obtain

$$
\frac{\partial^{2} w_{1}}{\partial r^{2}}{ }_{\left.\right|_{\Gamma_{L}}}=-\left.\frac{\partial^{2} w_{2}}{\partial z \partial r}\right|_{\Gamma_{L}}
$$


Let us differentiate the "divergence-free" condition with respect to $z$. It yields

$$
-\frac{\partial^{2} w_{2}}{\partial z \partial r}=\frac{\partial^{2} w_{1}}{\partial z^{2}}+\frac{1}{r} \frac{\partial w_{2}}{\partial z}
$$

The combination of equations (4.13), (4.14) and of the partial differential equation in $w_{1}$ (equation (2.9)) provides

$$
\frac{1}{\mu} \frac{\partial \widetilde{p}}{\left.\partial z\right|_{\Gamma_{L}}}=\left.2 \frac{\partial^{2} w_{1}}{\partial r^{2}}\right|_{\Gamma_{L}}+\left.\frac{2}{r} \frac{\partial w_{1}}{\partial r}\right|_{\Gamma_{L}} .
$$

Let us integrate this relation with respect to $r$, we obtain

$$
\frac{1}{\mu} \int_{\Gamma_{0}} \frac{\partial \widetilde{p}}{\partial z}=2 \int_{0}^{R_{0}} \frac{1}{r} \frac{\partial}{\partial r}\left(r \frac{\partial w_{1}}{\partial r}\right) \mathrm{d} r=2 R_{0} \frac{\partial w_{1}}{\partial r}\left(R_{0}, L\right)=-\left.2 R_{0} \frac{\partial w_{2}}{\partial z}\right|_{\Gamma_{a} \cap \Gamma_{L}}=0 .
$$

The combination of (4.12), (4.15) and the fact that $u_{0}^{\prime}\left(R_{0}\right) \neq 0$ provide a contradiction, which concludes the proof. $\square$

5. Conclusion. It is very common in shape optimization to get an existence result among domain verifying a kind of Lipschitz uniform regularity property called " $\varepsilon$ cone property" (see $[4,5,14])$. In this paper, the assumption that the optimal shape $\Omega$ is parametrized by a graph function $a$ has permitted to get an existence result among domains being less regular as usually (less than Lipschitz in particular). This issue being clarified, it would be now interesting to investigate the question of the general writing of the necessary first order optimality conditions. In particular, the differentiability of the criterion for domains having a low regularity (for instance, the case where $a$ belongs to $\left.W^{1,2}([0, L])\right)$ appears not so clear and we chose in this paper to write the first order optimality conditions with an additional regularity assumption on the boundary of the optimum. We were also in position to make a refined study of the shape optimization problem (2.21) and in particular to exploit the first order optimality condition to obtain a quasi explicit expression of the optimum. Nevertheless, our approach fails when an additional volume constraint is considered, as in problem (4.10). In every cases, a general existence theorem has been stated, but getting some qualitative properties on the optimum appeared highly difficult to lead in presence of a volume constraint.

We have now in mind a Lagrangian algorithm approach to compute optimal shapes numerically. As a consequence of Section 4.4, we will have to deal with nonsmooth constraints, treated with the introduction of subdifferentials, and with standard pointwise constraints. This will need a consequent analysis and will be done in a forthcoming work.

Let us mention that a first numerical study, where the same shape functional were minimized over three dimensional domains with a global volume constraint, has been led in [11].

\section{REFERENCES}

[1] G. Allaire Shape optimization by the homogenization method, Applied Mathematical Sciences, 146, SpringerVerlag, New York, 2002.

[2] G. Arumugam, O. Pironneau, On the problems of riblets as a drag reduction device, Optimal Control Appl. Methods 10 (1989), no. 2, 93-112.

[3] F. BOYER, P. FABRIE, Eléments d'analyse pour l'étude de quelques modèles d'écoulements de fluides visqueux incompressibles, Mathématiques et Applications, 52, Springer-SMAI, 2006

[4] D. Bucur, G. Buttazo, Variational methods in Shape Optimization Problems, Progress in Nonlinear Differential Equations and Their Applications, 65 Birkhäuser, Basel, Boston, 2005

[5] D. Chenais, On the existence of a solution in a domain identification problem, J. Math. Anal. Appl., 52 (1975), 189-289. 
[6] H. Choi, P. MoIn, J. KIM, Turbulent drag reduction: studies of feedback control and flow over riblets, CTR Rep. TF-55. Stanford, CA, 1992.

[7] M. DAuge Opérateur de Stokes dans des espaces de Sobolev à poids sur des domaines anguleux., Canad. J. Math. 34 (1982), no. 4, 853-882.

[8] M. DAuge Stationary Stokes and Navier-Stokes systems on two- or three-dimensional domains with corners. I. Linearized equations, SIAM J. Math. Anal., Vol. 20 1, (1989), 74-97.

[9] M. Delfour, J.P. ZolÉsio Shapes and geometries. Analysis, differential calculus, and optimization, Advances in Design and Control SIAM, Philadelphia, PA, 2001.

[10] J. C. De Los Reyes, R. GRIEsse State-constrained optimal control of the three-dimensional stationary NavierStokes equations, J. Math. Anal. Appl., Vol. 343 (1), (2008), 257-272.

[11] X. Dubois de la Sablonière, B. Mauroy, Y. Privat Shape minimization of the dissipated energy in dyadic trees, Discrete Contin. Dyn. Syst. Ser. B, Vol. 16 3, (2011), 767-799

[12] E. FeIREISL, Shape optimization in viscous compressible fluids, Appl. Math. Optim., 47 (2003), no. 1, 59-78.

[13] G. P. GALdi An Introduction to the Mathematical Theory of the Navier-Stokes Equations Volumes 1 and 2 , Springer Tracts in Natural Philosophy, Vol. 38, 1998.

[14] A. Henrot, M. Pierre, Variation et Optimisation de formes, coll. Mathématiques et Applications, vol. 48, Springer 2005.

[15] A. Henrot, Y. Privat, Une conduite cylindrique n'est pas optimale pour minimiser l'énergie dissipée par un fluide, C. R. Acad. Sci. Paris Sér. I Math, (2008).

[16] A. Henrot, Y. Privat, What is the optimal shape of a pipe?, Arch. Rat. Mech. Analysis, Vol. 196 (1), 281-302, (2010).

[17] M. Hinze, K. Kunisch, Second order methods for boundary control of the instationary Navier-Stokes system, ZAMM Z. Angew. Math. Mech., Vol. 84 (3), 171-187, (2004).

[18] M. Hinze, K. Kunisch, Second order methods for optimal control of time-dependent fluid flow, SIAM J. Control Optim., Vol. 40 (3), 925-946 (electronic), (2001).

[19] B. Kawohl, Rearrangements and convexity of level sets in PDE, Springer Lecture Notes in Math. 1150, p. 1-134. (1985).

[20] C. B. Morrey, Multiple Integrals in the Calculus of Variations, Springer, Berlin, 1966.

[21] J.-L. Lions, E. Magenes, Problèmes aux limites non homogènes et applications, Vol. I, II, III, Dunod, Paris, 1968.

[22] R. Lohner, Applied Computational Fluid Dynamics Techniques: An Introduction Based on Finite Element Methods., Chichester: Wiley 376 pp. 2001.

[23] B. Mauroy, M. Filoche, J.S. Andrade, B. Sapoval, Interplay between geometry and flow distribution in an airway tree, Physical Review Letters, 90, 2003, pp. 1-4.

[24] V. A. Kozlov, V. G. MAZ'YA, On "power-logarithmic" solutions to the Dirichlet problem for the Stokes system in a dihedral angle, Math. Methods Appl. Sci. 20 (1997), no. 4, 315-346.

[25] V. A. Kozlov, V. G. Maz'YA, The asymptotic behavior of solutions of the Navier-Stokes equations near the edges., Dokl. Akad. Nauk SSSR 210 (1973), 803-806.

[26] B. Mohammadi, O. Pironneau, Applied shape optimization for fluids, Clarendon Press, Oxford 2001.

[27] F. Murat, J. Simon, Sur le contrôle par un domaine géométrique, Publication du Laboratoire d'Analyse Numérique de l'Université Paris 6, 189, 1976.

[28] J. H Ortega, E. ZuazuA, Generic simplicity of the eigenvalues of the Stokes system in two space dimensions, Adv. Differential Equations, Vol. 6, no. 8, 987-1023, 2001. 1984.

[29] O. Pironneau, Optimal shape design for elliptic systems, Springer Series in Computational Physics, Springer, New York 1984.

[30] P. Plotnikov, J. Sokolowski, Domain Dependence of Solutions to Compressible Navier-Stokes Equations, SIAM J. Control Optim., Volume 45, Issue 4, pp. 1165-1197.

[31] J. Sokolowski Et J. P. Zolesio, Introduction to Shape Optimization Shape Sensitivity Analysis, Springer Series in Computational Mathematics, Vol. 16, Springer, Berlin 1992.

[32] L. Stupelis, Navier-Stokes equations in irregular domains, Mathematics and its Applications, $326,1995$.

[33] R. Temam Navier-Stokes Equations, North-Holland Pub. Company (1979), 500 pages. 